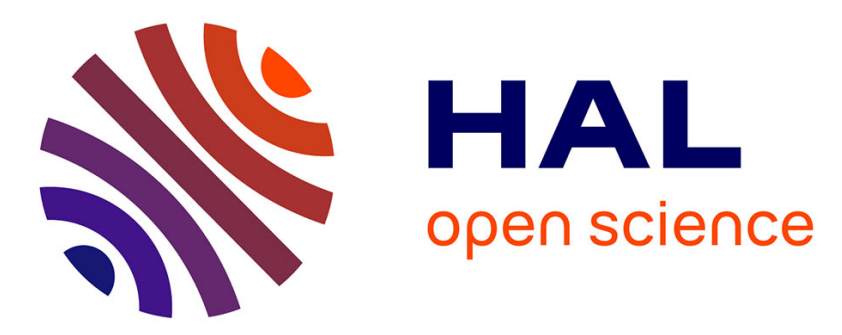

\title{
Shape optimal design problem with convective and radiative thermal transfer: Analysis and implementation
}

Denise Chenais, Jerome Monnier, Jean-Paul Vila

\section{To cite this version:}

Denise Chenais, Jerome Monnier, Jean-Paul Vila. Shape optimal design problem with convective and radiative thermal transfer: Analysis and implementation. Journal of Optimization Theory and Applications, 2001, 110 (1), pp.75-117. 10.1023/A:1017543529204 . inria-00256545

\section{HAL Id: inria-00256545 \\ https://hal.inria.fr/inria-00256545}

Submitted on 15 Feb 2008

HAL is a multi-disciplinary open access archive for the deposit and dissemination of scientific research documents, whether they are published or not. The documents may come from teaching and research institutions in France or abroad, or from public or private research centers.
L'archive ouverte pluridisciplinaire HAL, est destinée au dépôt et à la diffusion de documents scientifiques de niveau recherche, publiés ou non, émanant des établissements d'enseignement et de recherche français ou étrangers, des laboratoires publics ou privés. 


\title{
Shape Optimal Design Problem with Convective and Radiative Heat Transfer: Analysis and Implementation ${ }^{1}$
}

\author{
D. CHENAIS ${ }^{2}$, J. MONNIER ${ }^{3}$, J.P. VILA ${ }^{4}$
}

Communicated by K.K. Choi

1. This work and the software development were partially supported by the companies

Renault S.A. and Simulog S.A. The authors thank the referees for their helpful comments.

2. Professor, Université de Nice-Sophia Antipolis, Laboratoire de Mathématiques, Nice, France.

3. Assistant Professor, Institut National Polytechnique de Grenoble, Laboratoire de Modélisation et Calcul, Grenoble, France.

4. Professor, Institut National des Sciences Appliquées de Toulouse, Mathématiques pour l'Industrie et la Physique, Toulouse, France. 
Abstract. We present a study of an optimal design problem for a coupled system, consisting of a steady-state potential flow equation and a thermal equation taking into account radiative phenomena with multiple reflections. The state equation is a non-linear integro-differential system. We seek to minimize a cost function which depends on the temperature, with respect to the domain of the equations. First, we consider an optimal design problem in an abstract framework and, with the help of the classical adjoint state method, we give an expression of the cost function differential. Then, we apply this result in two spatial dimensions to the non-linear integro-differential system considered. We prove the differentiability of the cost function, we introduce the adjoint state equation, and we give an expression of its exact differential. Then, we discretize the equations by a finite element method and we use a gradient type algorithm to decrease the cost function. We present numerical results as applied to the automotive industry.

Key words. Shape sensitivity, optimal design, radiative heat transfer, elliptic partial differential equations, finite element method. 


\section{Introduction}

This paper deals with an optimal design problem in fluid mechanics and heat transfer which arises from a cooling problem in automobiles. The goal is to model a stationary air flow and heat exchanges under a car hood and then to optimize a hose shape in order to minimize a differentiable cost function which depends on the temperature. From the modeling standpoint, we choose in a first approach the simplest fluid model i.e. an incompressible potential flow, and we model heat transfer by convection, diffusion and radiation with multiple reflections. Hence, this problem is an optimal control problem governed by a non-linear integro-differential system in which the control variable is the domain of the equations (i.e. an optimal design problem). Numerically, we minimize the cost function with the help of an algorithm of descent.

We choose a continuous approach which means that, first we give an expression of the exact differential of the cost function, and then we discretize it using a finite element method.

This paper is organized as follows. In Section 2.1, we recall some results in optimal control of systems governed by a partial differential equation (linear or not)

in Banach spaces. We recall under which conditions, the state is differentiable with respect to the control variable, we introduce a classical adjoint state and we give an expression of the cost function differential (see Theorem 2.1). In Section 2.2, we consider an optimal design problem in an abstract framework. We recall the 
classical approach in which the space of admissible domains is a set of domains homeomorphic to a reference domain denoted by $\hat{\Omega}$. We consider $\hat{\Omega}$ as an open subset of $\mathbb{R}^{n}$ with a lipschitz boundary and bilipschitz homeomorphisms. It follows that the domain space is an open set. (This would not be true with $W^{1, \infty}$ homeomorphisms, Remark 2.1). Then, we define in a classical manner the derivative of a function with respect to the domain, using the method of transport. We show how a such optimal control problem fits into the framework of Section 2.1, and thanks to Theorem 2.1, we give an expression of the exact differential of the cost function (Corollary 2.1). Finally, we present derivatives of the integrals which appear later on in the cooling problem.

In Section 3, we present and study the direct problem. It is to model a stationary incompressible potential flow with heat transfer by conduction, convection and radiation. The model of radiation takes into account the emission, the reflection and the absorption of the radiant energy. The emitted and reflected radiation are diffusely distributed. The surfaces are assumed to be opaque and to behave like grey bodies i.e. the radiative exchanges do not depend on the wave length. In others respects, we assume that the surfaces are separated by a non participating media hence, the radiative transfer is apparent in the boundary conditions.

The full thermal model is an integro-differential system. The boundary condition of the partial differential equation is non linear, non local and non monotone. Its mathematical analysis has been written in 1 . Nevertheless, the coupling between the fluid model and the thermal model requires some regularity on the velocity field, 
hence we discuss the regularity of the solutions. In the twodimensional case (and under some assumptions), we obtain the existence and uniqueness of the solution to the coupled problem (Theorem 3.2). Moreover, we show that if the domain is three dimensional and if it has a re-entrant corner of $(2 \pi-\varepsilon)$ radians, for $\varepsilon>0$ given arbitrarily small, then the coupled system (potential flow - heat equation with a convective term) is not defined (in the classical weak sense).

In Section 4, we study the optimal design problem as applied to the automotive industry. The equations modeling the heat transfer under a car hood have been studied in Section 3 and the mathematical technic we use to treat the problem of optimal design have been presented in Section 2. Hence, in this section, we apply the analysis and technic of Section 2 to the direct problem of Section 3. We prove that if the thermal conductivity of the fluid is large enough (compared to the radiative terms) then the state of the system is differentiable with respect to the domain. It follows that the cost function is also differentiable. Then, we write the adjoint state equation and we give an expression for the exact differential (Proposition 4.1). The adjoint state equation is an integro-differential system like the state equation, but by construction it is linear. The equations of this system are solved in the reverse way compared to the state equation and each of them is the adjoint equation of the corresponding equation to the direct problem.

In Section 5, we discretize the state and the adjoint state equations using a finite element method. The numerical analysis of the thermal model has been writ- 
ten in 1 . We present some results of existence, uniqueness and convergence of the discrete solution of the coupled system (fluid model-thermal model) in theorems 5.1 and 5.2. Then, we discretize the expression of the exact differential of the cost function obtained in the previous section and we obtain the shape gradient.

We detail the implementation of the optimal design problem. By this end, we present the algorithms we use to solve the state equation, we detail how we discretize the control variable (i.e. the design variables) and we present a technic to compute the vector field of the perturbations of domain. Finally, we summarize the optimization process in Figure 5.

In Section 6, we present twodimensional numerical results of the air flow under a car hood. We obtain the same "optimal" shape from two different initial shapes. The goal of these numerical results is to prove that we can solve the initial cooling problem using the analysis and technic presented above. It is feasibility study. The software we wrote have to be more developed in order to obtain interesting results in an industrial and engineering point of view.

\section{Basic Concepts in Optimal Design}

The goal of this section is to recall basic concepts in shape optimum design. First, we recall some results in optimal control of systems governed by an elliptic partial differential equation (linear or not) in Banach spaces. We seek to minimize the cost function using an algorithm of descent. By this end, we give an expression of the exact differential of the cost function (Theorem 2.1). 
Then, we recall in Section 2.2 the framework of shape optimization using transformations of a reference domain and we explain how a problem of shape optimal design fits into the framework of Section 2.1.

\subsection{Optimal Control of Systems Governed by a Partial Dif- ferential Equation in Banach Spaces}

We consider the following optimal control problem. Let $\Lambda$ be a Banach space, we denote by $\Sigma$ the set of admissible controls, it is an open subset of $\Lambda$. Let $V$ be a reflexive Banach space, $a: \Sigma \times V \times V \rightarrow \mathbb{R}:(\sigma, y, z) \mapsto a(\sigma ; y, z)$ a functional which is linear, continuous with respect to $z$ and $l: \Sigma \times V \rightarrow \mathbb{R}:(\sigma, z) \mapsto l(\sigma ; z)$ a functional which is linear continuous with respect to $z$. Let us notice that the functional $a$ is not necessarily linear with respect to $y$. Let $J: \Sigma \times V \rightarrow \mathbb{R}$ : $(\sigma, z) \mapsto J(\sigma ; z)$ be a functional of class $C^{1}(\Sigma \times V)$. We consider the following state equation:

Find $y^{\sigma} \in V$ such that

$$
\forall z \in V, a\left(\sigma ; y^{\sigma}, z\right)=l(\sigma ; z)
$$

Problem (1) is supposed to be well posed (i.e. for all $\sigma \in \Sigma$ fixed, the state equation (1) has a unique solution $y^{\sigma} \in V$ which depends continuously on the data). We define the cost function by $j: \Sigma \rightarrow \mathbb{R}: \sigma \mapsto j(\sigma)=J\left(\sigma ; y^{\sigma}\right)$, where $y^{\sigma} \in V$ is the solution of (1). The control problem we consider is:

Find $\sigma^{\star} \in \Sigma$ such that

$$
j\left(\sigma^{\star}\right)=\min _{\sigma \in \Sigma} j(\sigma)
$$


We seek to solve this problem with the help of a gradient type algorithm. To this end, we give an expression of the exact differential of the cost function. In order to prove the differentiability of this cost function, we first prove the differentiability of the state $y^{\sigma}$ with respect to the control $\sigma$ then, we introduce in a classical manner an adjoint state which allows us to avoid computing the expression of the differential of $y^{\sigma}$ with respect to $\sigma$. Let us recall that we do not consider in this paper the problem of existence of a minimum $\sigma^{\star}$. The main result of this section is Theorem 2.1.

Let us introduce the operators $A: \Sigma \times V \rightarrow V^{\prime}$ and $L: \Sigma \rightarrow V^{\prime}$ such that $\forall z \in V,<A(\sigma ; y), z>_{V^{\prime} \times V}=a(\sigma ; y, z)$ and $<L(\sigma), z>_{V^{\prime} \times V}=l(\sigma ; z)$. Then, the state equation (1) is equivalent to:

$$
A\left(\sigma ; y^{\sigma}\right)=L(\sigma) \text { in } V^{\prime}
$$

For each $\sigma$, the operator $A(\sigma ;$.$) is a homeomorphism from V$ into $V^{\prime}$. We assume that $A \in C^{1}\left(\Sigma \times V ; V^{\prime}\right)$ and $L \in C^{1}\left(\Sigma ; V^{\prime}\right)$. We define the linearized problem as follows:

Find $w^{\sigma} \in V$ such that

$$
\frac{\partial A}{\partial y}\left(\sigma ; y^{\sigma}\right) \cdot w^{\sigma}=L(\sigma) \text { in } V^{\prime}
$$

It follows from the implicit function theorem applied to (2) that if the linearized problem (3) is well posed, then the state $y^{\sigma}$ is well defined and it is locally $C^{1}$ with respect to the control variable $\sigma$, i.e. there exists $\mathcal{V}(\sigma)$, a neighborhood of $\sigma$, such that Equation (2) defines a mapping $\sigma \mapsto y^{\sigma}: \mathcal{V}(\sigma) \subset \Sigma \rightarrow V$ which is $C^{1}$. 
Theorem 2.1 Assume that $A \in C^{1}\left(\Sigma \times V ; V^{\prime}\right), L \in C^{1}\left(\Sigma ; V^{\prime}\right)$, that the linearized problem (3) is well posed and that the observation function $J \in C^{1}(\Sigma \times V ; \mathbb{R})$. Then, there exists a neighborhood of $\sigma$ denoted by $\mathcal{V}(\sigma), \mathcal{V}(\sigma) \subset \Sigma \subset \Lambda$, such that the cost function $\tau \mapsto j(\tau): \mathcal{V}(\sigma) \rightarrow \mathbb{R}$ is of class $C^{1}$ and:

$$
\forall \delta \sigma \in \Lambda, \frac{d j}{d \sigma}(\sigma) \cdot \delta \sigma=\frac{\partial J}{\partial \sigma}\left(\sigma ; y^{\sigma}\right) \cdot \delta \sigma-\frac{\partial a}{\partial \sigma}\left(\sigma ; y^{\sigma}, p^{\sigma}\right) \cdot \delta \sigma+\frac{\partial l}{\partial \sigma}\left(\sigma ; p^{\sigma}\right) \cdot \delta \sigma
$$

where $y^{\sigma} \in V$ is the solution of the state equation (1), $p^{\sigma} \in V$ is the unique solution of the following adjoint state equation:

$$
\forall \delta y \in V, \quad \frac{\partial a}{\partial y}\left(\sigma ; y^{\sigma}, p^{\sigma}\right) . \delta y=\frac{\partial J}{\partial y}\left(\sigma ; y^{\sigma}\right) . \delta y
$$

Proof. We deduce from the implicit function theorem that there exists $\mathcal{V}(\sigma)$ such that the state $y^{\sigma}$ is $C^{1}$ in $\mathcal{V}(\sigma)$ and we assumed that $J$ is $C^{1}(\Sigma \times V)$. Therefore the cost function $j$ is $C^{1}$ from $\mathcal{V}(\sigma)$ into $\mathbb{R}$ and we have: $\frac{d j}{d \sigma}(\sigma) . \delta \sigma=\frac{\partial J}{\partial \sigma}\left(\sigma ; y^{\sigma}\right) \cdot \delta \sigma+$ $\frac{\partial J}{\partial y}\left(\sigma ; y^{\sigma}\right) \cdot\left(\frac{d y^{\sigma}}{d \sigma} . \delta \sigma\right)$. We write it as follows:

$$
\frac{d j}{d \sigma}(\sigma) . \delta \sigma=<\frac{\partial J}{\partial \sigma}\left(\sigma ; y^{\sigma}\right), \delta \sigma>_{\Lambda^{\prime} \times \Lambda}+<\frac{\partial J}{\partial y}\left(\sigma ; y^{\sigma}\right),\left(\frac{d y^{\sigma}}{d \sigma} \cdot \delta \sigma\right)>_{V^{\prime} \times V}
$$

Differentiating with respect to $\sigma$ the equation $A\left(\sigma ; y^{\sigma}\right)=L(\sigma)$, we obtain $\frac{\partial A}{\partial \sigma}\left(\sigma ; y^{\sigma}\right) \cdot \delta \sigma+\frac{\partial A}{\partial y}\left(\sigma ; y^{\sigma}\right) \cdot\left(\frac{d y^{\sigma}}{d \sigma} \cdot \delta \sigma\right)=\frac{d L}{d \sigma}(\sigma) . \delta \sigma$, which can be written:

$$
\begin{aligned}
& \forall v \in V, \quad<\frac{\partial A}{\partial \sigma}\left(\sigma ; y^{\sigma}\right) \cdot \delta \sigma, v>_{V^{\prime} \times V}+<\frac{\partial A}{\partial y}\left(\sigma ; y^{\sigma}\right) \cdot\left(\frac{d y^{\sigma}}{d \sigma} \cdot \delta \sigma\right), v>_{V^{\prime} \times V} \\
& -<\frac{d L}{d \sigma}(\sigma) \cdot \delta \sigma, v>_{V^{\prime} \times V}=0
\end{aligned}
$$

In addition, $V$ is a reflexive Banach space and the linearized problem is well posed i.e. $\frac{\partial A}{\partial y}\left(\sigma ; y^{\sigma}\right)$ is an isomorphism from $V$ into $V^{\prime}$, therefore its adjoint operator $\left(\frac{\partial A}{\partial y}\left(\sigma ; y^{\sigma}\right)\right)^{*}$ is also an isomorphism from $V$ into $V^{\prime}$, Ref. 2. Subtracting (6) to (5), 
we obtain:

$$
\begin{aligned}
\frac{d j}{d \sigma}(\sigma) . \delta \sigma= & <\frac{\partial J}{\partial \sigma}\left(\sigma ; y^{\sigma}\right), \delta \sigma>_{\Lambda^{\prime} \times \Lambda} \\
& +<\frac{\partial J}{\partial y}\left(\sigma ; y^{\sigma}\right)-\left(\frac{\partial A}{\partial y}\left(\sigma ; y^{\sigma}\right)\right)^{*} \cdot v,\left(\frac{d y^{\sigma}}{d \sigma} \cdot \delta \sigma\right)>_{V^{\prime} \times V} \\
& -<\frac{\partial A}{\partial \sigma}\left(\sigma ; y^{\sigma}\right) . \delta \sigma-\frac{d L}{d \sigma}(\sigma) . \delta \sigma, v>_{V^{\prime} \times V}
\end{aligned}
$$

Now, consider the solution $p^{\sigma} \in V$ of the following equation called the adjoint state equation:

$$
\forall z \in V, \quad<\left(\frac{\partial A}{\partial y}\left(\sigma ; y^{\sigma}\right)\right)^{*} \cdot p^{\sigma}, z>_{V^{\prime} \times V}=<\frac{\partial J}{\partial y}\left(\sigma ; y^{\sigma}\right), z>_{V^{\prime} \times V}
$$

(Let us notice that since the adjoint operator $\left(\frac{\partial A}{\partial y}\left(\sigma ; y^{\sigma}\right)\right)^{*}$ is an isomorphism from $V$ into $V^{\prime}$, the adjoint state $p^{\sigma} \in V$ exists and is unique). Choosing $v=p^{\sigma}$ in (7), we obtain:

$$
\frac{d j}{d \sigma}(\sigma) . \delta \sigma=<\frac{\partial J}{\partial \sigma}\left(\sigma ; y^{\sigma}\right), \delta \sigma>_{\Lambda^{\prime} \times \Lambda}-<\frac{\partial A}{\partial \sigma}\left(\sigma ; y^{\sigma}\right) . \delta \sigma-\frac{d L}{d \sigma}(\sigma) . \delta \sigma, p^{\sigma}>_{V^{\prime} \times V}
$$

Finally, we obtain:

$$
\frac{d j}{d \sigma}(\sigma) \cdot \delta \sigma=\frac{\partial J}{\partial \sigma}\left(\sigma ; y^{\sigma}\right) \cdot \delta \sigma-\frac{\partial a}{\partial \sigma}\left(\sigma ; y^{\sigma}, p^{\sigma}\right) \cdot \delta \sigma+\frac{\partial l}{\partial \sigma}\left(\sigma ; p^{\sigma}\right) \cdot \delta \sigma
$$

\subsection{Optimal Design}

In this section, we recall the framework of shape optimization using transformations of a reference domain. The problem is the following. Let $\mathcal{D}$ be a set of admissible domains and $j$ be a cost function $j: \mathcal{D} \rightarrow \mathbb{R}: \omega \mapsto j(\omega)$. We seek $\omega^{\star} \in \mathcal{D}$ such that $j\left(\omega^{\star}\right)=\min _{(\omega \in \mathcal{D})} j(\omega)$. We want to solve this problem with the help of a gradient type algorithm.

The mathematical difficulty of this problem, which is classical, is the following. On 
one hand, the set of domains $\mathcal{D}$ is not a vectorial space and on the other hand, we want to give an expression of the differential of the cost function $j(\omega)$ in order to use a gradient type algorithm. To avoid this difficulty and following Ref. 3, we recall the definition of admissible domains and we define in a classical manner the derivative of a real valued function with respect to the domain. Then, we are able to give an expression of the differential of the cost function with intent to call a gradient type algorithm.

In Section 2.2.3, we consider an optimal design problem and using the previous definitions, we explain how such a problem fits into the framework of Section 2.1. The main result of this section is Corollary 2.1 and most of the following results derive from Refs. 3 and 4. The reader may also consult Refs. 5-12.

\subsubsection{Definition of the Space of Admissible Domains}

We define in a classical manner the space of admissible domains as a set of domains which are homeomorphic to a reference domain denoted by $\hat{\Omega}$. In an optimal design problem, the regularity of these homeomorphisms depends on the order of the partial differential equation of which the state is the solution.

Framework for First and Second Order Problems Let $\hat{\Omega}$ be an open subset of $\mathbb{R}^{n}$ with a lipschitz boundary (see e.g Ref. 14), we consider:

$\operatorname{Lip}\left(\hat{\Omega}, \mathbb{I}^{n}\right)=\left\{\hat{T}: \hat{\Omega} \rightarrow \mathbb{I R}^{n} ; \exists k\right.$ such that $\left.\forall(\hat{x}, \hat{y}) \in \hat{\Omega} \times \hat{\Omega},\|\hat{T}(\hat{x})-\hat{T}(\hat{y})\| \leq k\|\hat{x}-\hat{y}\|\right\}$

equipped with the norm $\|\hat{T}\|_{\text {Lip }}=\sup _{\hat{x} \in \hat{\Omega}}\|\hat{T}(\hat{x})\|+\sup _{(\hat{x}, \hat{y}) \in \hat{\Omega} \times \hat{\Omega}} \frac{\|\hat{T}(\hat{x})-\hat{T}(\hat{y})\|}{\|\hat{x}-\hat{y}\|}$.

Then we consider the set $\hat{\mathcal{T}}^{1}$ of bilipischtzian homeomorphisms defined from $\hat{\Omega}$ into 
$\mathbb{R}^{n}$ :

$\hat{\mathcal{T}}^{1}=\left\{\hat{T}\right.$ bijection of $\hat{\Omega}$ onto $\left.\hat{T}(\hat{\Omega}) ; \hat{T} \in \operatorname{Lip}\left(\hat{\Omega}, \mathbb{R}^{n}\right), \hat{T}^{-1} \in \operatorname{Lip}\left(\hat{T}(\hat{\Omega}), \mathbb{R}^{n}\right)\right\}$

Lemma 2.1 $\hat{\mathcal{T}}^{1}$ is an open set for the topology of $\operatorname{Lip}\left(\hat{\Omega}, \mathbb{I}^{n}\right)$.

Proof. Let $\hat{T} \in \hat{\mathcal{T}}^{1}$, we denote by $\hat{B}(0,1)$ the unit ball of $\operatorname{Lip}\left(\hat{\Omega}, \mathbb{I}^{n}\right)$. For all $\hat{U} \in \hat{B}(0,1)$, for all $\varepsilon>0,(\hat{T}+\varepsilon \hat{U}) \in \operatorname{Lip}\left(\hat{\Omega}, \mathbb{R}^{n}\right)$. Moreover, as $\hat{T} \in \hat{\mathcal{T}}^{1}$, there exist $k_{1}>0$ and $k_{2}>0$ such that:

$$
\forall \hat{x} \in \hat{\Omega}, \forall \hat{y} \in \hat{\Omega}, \quad k_{1}\|\hat{x}-\hat{y}\| \leq\|\hat{T}(\hat{x})-\hat{T}(\hat{y})\| \leq k_{2}\|\hat{x}-\hat{y}\|
$$

and

$$
\begin{aligned}
\|(\hat{T}+\varepsilon \hat{U})(\hat{x})-(\hat{T}+\varepsilon \hat{U})(\hat{y})\| & \geq\|\hat{T}(\hat{x})-\hat{T}(\hat{y})\|-\varepsilon\|\hat{U}(\hat{x})-\hat{U}(\hat{y})\| \\
& \geq\left(k_{1}-\varepsilon\right)\|\hat{x}-\hat{y}\|=k\|\hat{x}-\hat{y}\|
\end{aligned}
$$

So, for $\varepsilon<k_{1},(\hat{T}+\varepsilon \hat{U})^{-1}$ exists and is lipschitz continuous. This completes the proof.

Definition 2.1 Let $\hat{\Omega}$ be a bounded open subset of $\mathbb{R}^{n}$ with a lipschitz boundary, we define the admissible domains space $\mathcal{D}$ as follows:

$$
\mathcal{D}=\left\{\omega=\hat{T}(\hat{\Omega}) ; \hat{T} \in \hat{\mathcal{T}}^{1}\right\}
$$

Remark 2.1 In this paper, we consider lipschitz homeomorphisms from $\hat{\Omega}$ into $\mathbb{R}^{n}$ and not homeomorphisms of class $W^{1, \infty}$. This slightly differs from the usual definition of $\mathcal{D}$, see Ref. 3. As a matter of fact, the image of a regular bounded open subset of $I R^{n}$ by a $W^{1, \infty}$ homeomorphism is not necessarily an open subset of $I R^{n}$ 
with a lipschitz boundary.

Let us give an example (see Figure 1). Let $\hat{\Omega}$ be the open set of $\mathbb{R}^{2}$ defined by: $\hat{\Omega}=\hat{\Omega}_{1} \cup \hat{\Omega}_{2} \cup \hat{\Omega}_{3} \cup \hat{\Omega}_{4}$ with $\hat{\Omega}_{1}=\left\{\left(x_{1}, x_{2}\right) /-2<x_{1}<0, \quad-2<x_{2}<1\right\}$, $\hat{\Omega}_{2}=\left\{\left(x_{1}, x_{2}\right) / 0 \leq x_{1} \leq 1,-2<x_{2}<-1\right\}, \hat{\Omega}_{3}=\left\{\left(x_{1}, x_{2}\right) / 1<x_{1}<2,-2<\right.$ $\left.x_{2}<1\right\}$, and $\hat{\Omega}_{4}=\left\{\left(x_{1}, x_{2}\right) / \frac{1}{2}<x_{1} \leq 1,0<x_{2}<1\right\} . \hat{\Omega}$ is an open set with a lipschitz boundary.

We define $\hat{T}$ from $\hat{\Omega}$ into $\mathbb{R}^{2}$ by:

$$
\hat{T}\left(\hat{x}_{1}, \hat{x}_{2}\right)=\mid \begin{array}{ll}
\left(\hat{x}_{1}, \hat{x}_{2}\right) & \text { in } \hat{\Omega}_{1} \cup \hat{\Omega}_{2} \cup \hat{\Omega}_{3} \\
\left(2 \hat{x}_{1}-1, \hat{x}_{2}\right) & \text { in } \hat{\Omega}_{4}
\end{array}
$$

Then, $\hat{T}$ is a homeomorphism of class $W^{1, \infty}$ from $\hat{\Omega}$ onto its image, and its inverse $\hat{T}^{-1}$ is $W^{1, \infty}$. On the other hand, $\hat{T}(\hat{\Omega})$ is not an open set with a lipschitz boundary and $\hat{T}^{-1}$ is not a lipschitz mapping from $\hat{T}(\hat{\Omega})$ into $\hat{\Omega}$.

Indeed, let us recall that if $\hat{\Omega}$ is an open set with a lipschitz boundary then $\hat{T}$ belongs to $W^{1, \infty}\left(\hat{\Omega}, \mathbb{R}^{n}\right)$ if and only if $\hat{T}$ belongs to $\operatorname{Lip}\left(\hat{\Omega}, \mathbb{R}^{n}\right)$. On the other hand, if $\hat{\Omega}$ is less regular, then a mapping belonging to $W^{1, \infty}\left(\hat{\Omega}, \mathbb{R}^{n}\right)$ is not necessarily lipschitz.

Finally, the image of an open set with a lipschitz boundary by a Bi-lipschitz homeomorphism is not necessarily lipschitz. Nevertheless, if the mapping is close enough to the identity $I$, it is still an open set with a lipschitz boundary [M. Zerner, private communication]. 


\subsubsection{Derivative of a Real Valued Function with Respect to the Domain}

Let $\hat{\Omega} \subset \mathbb{R}^{n}$ be a bounded lipschitz open set. For $\hat{T}_{0} \in \hat{\mathcal{T}}^{1}$, we let $\Omega=\hat{T}_{0}(\hat{\Omega})$ (see Figure 2) and we define $\mathcal{T}^{1}$ the space of Bi-lipschitz homeomorphisms defined on $\Omega$ by:

$$
\mathcal{T}^{1}=\left\{T=\hat{T} \circ \hat{T}_{0}^{-1}, \hat{T} \in \hat{\mathcal{T}}^{1}\right\}
$$

Let $T \in \mathcal{T}^{1}$, we define $V \in \operatorname{Lip}\left(\Omega, \mathbb{R}^{n}\right)$ by $V=T-I$ (I denotes the identity of $\left.\mathbb{R}^{n}\right)$. For a given cost function $j$, we define $\hat{\jmath}$ by:

$$
\hat{\jmath}: \hat{\mathcal{T}}^{1} \rightarrow \mathbb{R}: \hat{T} \mapsto \hat{\jmath}(\hat{T})=j(\hat{T}(\hat{\Omega}))
$$

Definition 2.2 The mapping $j$ is said to be differentiable from $\mathcal{D}$ into $\mathbb{R}$ at the point $\Omega \in \mathcal{D}$ if and only if the mapping $\hat{\jmath}: \hat{\mathcal{T}}^{1} \rightarrow \mathbb{R}$ is Fréchet-differentiable at any point $\hat{T}_{0}$ such that $\hat{T}_{0}(\hat{\Omega})=\Omega$. Moreover, if $j$ is differentiable, we define and we define:

$$
\frac{d j}{d \omega}(\Omega) \cdot V=\frac{d \hat{\jmath}}{d \hat{T}}\left(\hat{T}_{0}\right) \cdot\left(V \circ \hat{T}_{0}\right), \forall V \in \operatorname{Lip}\left(\Omega, \mathbb{R}^{n}\right)
$$

This definition is legitimate because the differential does not depend on the choice of $\hat{T}_{0}$ such that $\hat{T}_{0}(\hat{\Omega})=\Omega$, Ref. 11 .

Let us also recall that if $\Omega$ is a connected open set, the derivative of the cost function in the direction $V$ only depends on the value of $V$ on the boundary (see Ref. 3, Theorem 3.1). In addition, if $\Omega$ is a bounded open set of class $C^{1}$, the derivative of the cost function in the direction $V$ depends only on the normal component of $V$ on $\partial \Omega$ (see Ref. 3, Theorem 3.1). 
Now, we give a "local expression" of the derivative in which everything is expressed on $\Omega$ instead of $\hat{\Omega}$. We will see later that it is very useful. By this end, let us define $\bar{\jmath}: \mathcal{T}^{1} \rightarrow \mathbb{R}: T \mapsto \bar{\jmath}(T)=\hat{\jmath}\left(T \circ \hat{T}_{0}\right)(=j(\omega))$. It is easy to prove the following result (see Ref. 11):

Proposition 2.1 The mapping $\bar{\jmath}$ is differentiable at the point $T=I$ in the direction $\mathrm{V}$ if and only if the mapping $\hat{\jmath}$ is differentiable at the point $\hat{T}=\hat{T}_{0}$ in the direction $\left(V \circ \hat{T}_{0}\right)$, and:

$$
\frac{d \bar{\jmath}}{d T}(I) \cdot V=\frac{d \hat{\jmath}}{d \hat{T}}\left(\hat{T}_{0}\right) \cdot\left(V \circ \hat{T}_{0}\right), \quad \forall V \in \operatorname{Lip}\left(\Omega, \mathbb{R}^{n}\right)
$$

Remark 2.2 $\hat{\Omega}$ is a fixed reference domain. Let $\omega=\hat{T}(\hat{\Omega})$, we have $j(\omega)=\hat{j}(\hat{T})$. The derivative of $j$ with respect to $\omega$ is by definition, the derivative of $\hat{\jmath}$ with respect to $\hat{T}$. Hence, minimizing $j$ in $\mathcal{D}$ is equivalent to minimize $\hat{\jmath}$ in $\hat{\mathcal{T}}^{1}$ :

$$
\min _{\omega \in \mathcal{D}} j(\omega) \Longleftrightarrow \min _{\hat{T} \in \hat{\mathcal{T}}^{1}} \hat{\jmath}(\hat{T})
$$

In an optimization algorithm which builds a sequence $\left(\Omega_{n}\right)_{n \in N N}$ and thanks to Proposition 2.1, at each step $n$ it is legitimate to consider $\Omega_{n}$ as the reference domain, by differentiating with respect to $T$, at $T=I$.

\subsubsection{Optimal Design Problem}

In this section like in Section 2.1, we consider an optimal control problem governed by an elliptic partial differential equation but now the control variable is the shape of the domain of the equation. Let us recall that a set of domains is not a vectorial space and we avoid this classical difficulty by using the technic presented in sections 


\subsection{1 and 2.2.2.}

We consider the space of admissible domains $\mathcal{D}$ defined by (8). For all $\omega \in \mathcal{D}$, we consider a Hilbert space $V(\omega)$, a functional $a_{\omega}: V(\omega) \times V(\omega) \rightarrow \mathbb{R}:(y, z) \mapsto$ $a_{\omega}(y, z)$ linear and continuous with respect to $z$, a functional $l_{\omega}: V(\omega) \rightarrow \mathbb{R}: z \mapsto$ $l_{\omega}(z)$ linear and continuous, an observation function $J_{\omega}: V(\omega) \rightarrow \mathbb{R}: z \mapsto J_{\omega}(z)$ of class $C^{1}$ and a cost function $j: \mathcal{D} \rightarrow \mathbb{R} ; \omega \mapsto j(\omega)=J_{\omega}\left(y^{\omega}\right)$, where $y^{\omega}$ is the unique solution of the following state equation:

$$
y^{\omega} \in V(\omega): \forall z \in V(\omega), a_{\omega}\left(y^{\omega}, z\right)=l_{\omega}(z)
$$

which is supposed to be well posed. We seek to solve the following problem:

Find $\omega^{\star} \in \mathcal{D}$ such that

$$
j\left(\omega^{\star}\right)=\min _{\mathcal{D}} j(\omega)
$$

Transport of Equations In order to compute the differential of the cost function $j$, we have to differentiate the state equation (10) (see Theorem 2.1); however this is not straightforward. According to Section 2.2.2, we transport the equations to $\Omega=T^{-1}(\omega)$ which is considered as the reference domain (see Remark 2.2). We assume:

Assumption 2.1 The mapping $z \in V(T(\Omega)) \mapsto z \circ T \in V(\Omega)$ is an isomorphism.

For any $y, z \in V(\omega)$, we let:

$$
\bar{a}(T ; \bar{y}, \bar{z})=a_{T(\Omega)}\left(\bar{y} \circ T^{-1}, \bar{z} \circ T^{-1}\right)=a_{\omega}(y, z)
$$


with $\omega=T(\Omega), y=\bar{y} \circ T^{-1}$ and $z=\bar{z} \circ T^{-1}, \forall y, z \in V(\omega)$ (see Fig. 6). Also, we let:

$$
\begin{aligned}
& \bar{l}(T ; \bar{z})=l_{T(\Omega)}\left(\bar{z} \circ T^{-1}\right)=l_{\omega}(z) \\
& \bar{J}(T ; \bar{y})=J_{T(\Omega)}\left(\bar{y} \circ T^{-1}\right)=J_{\omega}(y) .
\end{aligned}
$$

With Assumption 2.1, the state equation (10) is equivalent to

$$
\bar{y}^{T} \in V(\Omega): \forall \bar{z} \in V(\Omega), \bar{a}\left(T ; \bar{y}^{T}, \bar{z}\right)=\bar{l}(T ; \bar{z})
$$

where $\bar{y}^{T}=y^{\omega} \circ T$. We write (11) as follows:

$$
\bar{y}^{T} \in V(\Omega) ; \bar{A}\left(T ; \bar{y}^{T}\right)=\bar{L}(T) \text { in }(V(\Omega))^{\prime}
$$

where the operators $\bar{A}$ and $\bar{L}$ are defined by $<\bar{A}\left(T ; \bar{y}^{T}\right), \bar{z}>_{(V(\Omega))^{\prime} \times V(\Omega)}=\bar{a}\left(T ; \bar{y}^{T}, \bar{z}\right)$ and $<\bar{L}(T), \bar{z}>_{V^{\prime} \times V}=\bar{l}(T ; \bar{z})$ for all $\bar{z}$ in $V(\Omega)$.

We write $\bar{\jmath}(T)=\bar{J}\left(T ; \bar{y}^{T}\right)=j(\omega)$. The minimization problem we solve is now: Find $T^{\star} \in \mathcal{T}^{1}$ such that

$$
\bar{\jmath}\left(T^{\star}\right)=\min _{T \in \mathcal{T}^{1}} \bar{\jmath}(T)
$$

This problem fits in the general setting of Section 2.1 and we apply the same technique. Let us introduce the following linearized problem:

Find $w^{\omega} \in V(\omega)$ such that

$$
\forall z \in V(\omega), \frac{\partial a_{\omega}}{\partial y}\left(y^{\omega}, z\right) \cdot w^{\omega}=l_{\omega}(z)
$$

If this problem (13) is well posed, then thanks to Assumption 2.1, then the corresponding transported equation is well posed as well. It follows from the implicit function theorem applied to (12) that if $\bar{A} \in C^{1}\left(\mathcal{T}^{1} \times V(\Omega) ;(V(\Omega))^{\prime}\right)$ and 
$\bar{L} \in C^{1}\left(\mathcal{T}^{1} ;(V(\Omega))^{\prime}\right)$ then the state $\bar{y}^{T}$ is locally $C^{1}$ with respect to the control variable $T$ (namely, there exists $\mathcal{V}(T)$, a neighborhood of $T$, such that $T \mapsto \bar{y}^{T}$ is a $C^{1}$ mapping from $\mathcal{V}(T) \subset \mathcal{T}^{1}$ into $V(\Omega)$ ).

Let us notice that if $T=I$ then $\omega=\Omega, \bar{y}=y$ and $\bar{z}=z$, and we can derive directly from Theorem 2.1 the following result (see Ref. 11 for the proof).

Corollary 2.1 Assume that the operator $\bar{A}$ belongs to $C^{1}\left(\mathcal{T}^{1} \times V(\Omega) ;(V(\Omega))^{\prime}\right)$ and $\bar{L}$ belongs to $C^{1}\left(\mathcal{T}^{1} ;(V(\Omega))^{\prime}\right)$, the linearized problem (19) is well posed and the observation function $\bar{J}$ belongs to $C^{1}\left(\mathcal{T}^{1} \times V(\Omega) ; \mathbb{R}\right)$. Then there exists $\mathcal{V}(T)$, a neighborhood of $T$, such that the cost function $\omega \mapsto j(\omega)=J_{\omega}\left(y^{\omega}\right): \mathcal{D} \rightarrow \mathbb{R}$ belongs to $C^{1}$ for all $\omega=T(\Omega), T \in \mathcal{V}(T) \subset \mathcal{T}^{1}$. And for all $V \in \operatorname{Lip}\left(\Omega, \mathbb{R}^{n}\right)$,

$$
\frac{d j}{d \omega}(\Omega) \cdot V=\frac{\partial \bar{J}}{\partial T}\left(I ; y^{\Omega}\right) \cdot V-\frac{\partial \bar{a}}{\partial T}\left(I ; y^{\Omega}, p^{\Omega}\right) \cdot V+\frac{\partial \bar{l}}{\partial T}\left(I ; p^{\Omega}\right) \cdot V
$$

where $y^{\Omega} \in V(\Omega)$ is the solution of the state equation:

$$
\forall z \in V(\Omega), a_{\Omega}\left(y^{\Omega}, z\right)=l_{\Omega}(z)
$$

and $p^{\Omega} \in V(\Omega)$ is the unique solution of the adjoint state equation:

$$
\forall \delta y \in V(\Omega), \frac{\partial a_{\Omega}}{\partial y}\left(y^{\Omega}, p^{\Omega}\right) \cdot \delta y=\frac{\partial J_{\Omega}}{\partial y}\left(y^{\Omega}\right) \cdot \delta y
$$

Remark 2.3 Let us recall that in the adjoint state method, the expression of the differential of the cost function contains only partial derivatives with respect to $T$. When $y$ and $z \in V(\Omega)$ are fixed, the "partial derivatives" with respect to the domain at $\Omega$ in the direction $V$ of the mappings $J_{\omega}(y), a_{\omega}(y, z)$ and $l_{\omega}(z)$ are defined in a 
classical manner as follows (see e.g. Refs. 6, 5, 8, 9, 10, 11):

$\frac{\partial J_{\Omega}}{\partial \omega}(y) \cdot V=\frac{\partial \bar{J}}{\partial T}(I ; y) \cdot V, \frac{\partial a_{\Omega}}{\partial \omega}(y, z) \cdot V=\frac{\partial \bar{a}}{\partial T}(I ; y, z) \cdot V, \frac{\partial l_{\Omega}}{\partial \omega}(z) \cdot V=\frac{\partial \bar{l}}{\partial T}(I ; z) \cdot V$.

Then, we have the "classical" formula of the gradient: for all $V \in \operatorname{Lip}\left(\Omega, \mathbb{R}^{n}\right)$,

$$
\frac{d j}{d \omega}(\Omega) \cdot V=\frac{\partial J_{\Omega}}{\partial \omega}\left(y^{\Omega}\right) \cdot V-\frac{\partial a_{\Omega}}{\partial \omega}\left(y^{\Omega}, p^{\Omega}\right) \cdot V+\frac{\partial l_{\Omega}}{\partial \omega}\left(p^{\Omega}\right) \cdot V
$$

where $y^{\Omega} \in V(\Omega)$ and $p^{\Omega} \in V(\Omega)$ are respectively the solutions of the state equation (15) and of the adjoint state equation (16).

Some Examples of Derivatives We considered above the weak formulation of the state equation. Hence, in order to compute the differential of the cost function, we need to differentiate integrals formulas (or volume or surface) with respect to their domain of integration. In the proposition below, we give the derivatives of the integral formulas that we need in the sequel in order to solve the optimal design problem for the fluid-heat coupled system .

Let us notice that a bounded open set $\Omega$ with a lipschitz boundary has an external normal vector almost everywhere on its boundary, and we can define a surface integral on its boundary. In addition, if $T$ belongs to $\operatorname{Lip}\left(\Omega, \mathbb{R}^{n}\right)$ then $\left.T\right|_{\partial \Omega}$ belongs to $W^{1, \infty}\left(\partial \Omega, \mathbb{I}^{n}\right)$. Let us notice too, that the spaces $H^{1}(\omega)$ and $L^{p}(\omega), 1 \leq p<\infty$, satisfy Assumption 2.1.

Proposition 2.2 We denote by $\Omega$ an element of $\mathcal{D}$.

i) Consider $J_{\omega}(y)=\int_{\omega}|y|^{p} d x, 1<p<\infty$ where $y \in L^{p}(\omega)$. Then for all $V \in$ $\operatorname{Lip}\left(\Omega, \mathbb{R}^{n}\right)$

$$
\frac{\partial J_{\Omega}}{\partial \omega}(y) \cdot V=\int_{\Omega}|y|^{p} d i v V d x
$$


ii) Consider $a_{\omega}(u, z)=\int_{\omega}\langle u, \nabla z\rangle d x$ where $u \in\left(L^{2}(\omega)\right)^{n}, z \in H^{1}(\omega)$ and $<., .>$ denotes the inner product in $\mathbb{R}^{n}$. For all $V \in \operatorname{Lip}\left(\Omega, \mathbb{R}^{n}\right)$,

$$
\frac{\partial a_{\Omega}}{\partial \omega}(u, z) \cdot V=\int_{\Omega}\langle u, \nabla z\rangle \operatorname{div} V d x-\int_{\Omega}\langle D V . u, \nabla z\rangle d x
$$

iii) Consider $a_{\omega}(y, z)=\int_{\omega}\langle\nabla y, \nabla z\rangle d x$ where $y, z \in H^{1}(\omega)$. For all $V \in$ $\operatorname{Lip}\left(\Omega, \mathbb{R}^{n}\right)$

$\frac{\partial a_{\Omega}}{\partial \omega}(y, z) \cdot V=\int_{\Omega}<\nabla y, \nabla z>\operatorname{div} V d x-\int_{\Omega}<\left(D V+{ }^{t} D V\right) \cdot \nabla y, \nabla z>d x$

iv) Consider $J_{\omega}(y)=\int_{\partial \omega}|y|^{p} d s(x)$ where $y \in L^{p}(\partial \omega)$. For all $V \in \operatorname{Lip}\left(\Omega, \mathbb{R}^{n}\right)$,

$$
\frac{\partial J_{\Omega}}{\partial \omega}(y) \cdot V=\int_{\partial \Omega}|y|^{p} \operatorname{div}_{\Gamma} V d s(x),
$$

where $\operatorname{div}_{\Gamma} V$ is the tangential divergence $\left(\operatorname{div}_{\Gamma} V=\operatorname{div} V-\langle D V \cdot n, n\rangle=\langle\tau, D V \cdot \tau\rangle\right)$, $n$ is the unit external normal vector to the boundary, $\tau$ is the unit tangent vector such that $(\tau, n)$ is direct.

v) Consider $J_{\omega}(\phi)=\int_{\partial \omega} \int_{\partial \omega} \phi(x, y) d s(x) d s(y)$ where $\phi \in L^{1}(\partial \omega \times \partial \omega)$. For all $V \in \operatorname{Lip}\left(\Omega, \mathbb{R}^{n}\right)$

$$
\frac{\partial J_{\Omega}}{\partial \omega}(\phi) \cdot V=\int_{\partial \Omega} \int_{\partial \Omega} \phi(x, y)\left[\operatorname{div}_{\Gamma}(V)(x)+\operatorname{div}_{\Gamma}(V)(y)\right] d s(x) d s(y)
$$

Proof. The computation method has been described in the previous sections: we transport the mappings to the reference domain $\Omega$; then, by definition, the partial derivative with respect to the domain is the partial derivative of the transported mappings with respect to $T$ (see remark 2.3). We refer to Ref. 11 for the detailed computations. 


\section{Direct Problem}

In the previous section, we presented mathematical technic of optimal design in an abstract framework. In Section 4, we apply these technic to the cooling problem under a car hood. In the present section, we present the heat transfer model we consider and we write its analysis (Theorem 3.2). Let us recall that we call "direct problem" the equations which model the heat transfer with the domain $\omega$ given.

We consider an air flow under a car hood. Parts under the hood are schematized as indicated in Figure 4. We assume that the fluid flow is a stationary incompressible potential flow and we model heat transfer by convection, diffusion and radiation.

The fluid velocity $\vec{u}$ derives from a potential $\psi$, so we have $\vec{u}=\vec{\nabla} \psi$. Then, the continuity equation gives $\Delta \psi=0$ in $\omega$. The fluid boundary conditions are as follows. The potential is known on the exit side (see Figure 4), the normal velocity of the flow is known on the air entrance and is zero everywhere else on the boundary. We denote by $\gamma_{d}^{\psi}$ the boundary part where the potential is known (Dirichlet's condition) and by $\gamma_{n}^{\psi}$ the boundary part where $\nabla \psi \cdot n$ is known (Neumann's condition). We have $\gamma_{d}^{\psi} \cup \gamma_{n}^{\psi}=\partial \omega$ and $m e s\left(\gamma_{d}^{\psi}\right)>0$.

We denote by $\theta$ the fluid temperature. The conservation of energy equation gives:

$$
-\lambda \Delta \theta+\rho C_{p} \vec{u} \cdot \vec{\nabla} \theta=0 \text { in } \omega
$$

where $\lambda, \rho$ and $C_{p}$ are respectively the thermal conductivity, the density and the specific heat (at constant pressure) of the fluid.

We choose a model of radiation which takes into account the emission, the reflection and the absorption of the radiant energy. The emitted and reflected radiation are 
diffusely distributed. The surfaces are assumed to be opaque and to behave like grey bodies i.e. the radiative exchanges do not depend on the wave length. In addition, we assume that the surfaces are separated by a non participating media, hence the radiative heat transfer is apparent in the boundary conditions and is described by the radiosity (see e.g. Ref. 13). The radiosity is the radiant energy which flows away from a surface, it is denoted by $w$ and it is solution of the following Fredholm integral equation of the second kind (called the radiosity equation, see Ref. 13):

$$
w(x)=(1-\varepsilon(x)) \int_{\partial \omega} \phi(x, y) w(y) d s(y)+\varepsilon(x) \sigma \theta^{4}(x) \quad \text { on } \partial \omega
$$

$\sigma$ is the Stephan-Boltzmann constant. The function $\varepsilon$ is the surface emittance and satisfies: $0<\varepsilon_{0} \leq \varepsilon(x) \leq \varepsilon_{1} \leq 1$ on $\partial \omega$. The kernel $\phi \in L^{1}(\partial \omega \times \partial \omega)$ is the angle factor, it is positive, symmetric and satisfies:

$$
\int_{\partial \omega} \phi(x, y) d s(x)=1
$$

We refer for instance to Ref. 13 for the expression of the angle factor $\phi(x, y)$. Finally, the boundary conditions of the thermal partial differential equation are as follows. Temperatures are imposed on the air entrance, on the engine block and on the exhaust pipe. We consider on the external surface, on the hose, on the screen and on the battery, heat transfer by radiation and convection as follows. We denote by $\gamma_{f}^{\theta}$ this boundary part where the heat transfer is radiative and convective. The surface emittance $\varepsilon$ is assumed to be strictly lower than 1 on $\gamma_{f}^{\theta}$. If we denote by $h$ the thermal transfer coefficient and by $\theta_{0}$ the outside temperature, it follows from Fourier's law that:

$$
-\lambda \vec{\nabla} \theta \cdot \vec{n}=h\left(\theta-\theta_{0}\right)+\frac{\varepsilon}{1-\varepsilon}\left(\sigma \theta^{4}-w\right) \quad \text { on } \gamma_{f}^{\theta}
$$


where $w$ is solution of $(17)$ and $n$ is the external normal to $\partial \omega$. For a sake of simplicity, we assume that the thermal transfer coefficient $h$ does not depend on the fluid velocity $\vec{u}$, it is a constant. Concerning the air exit, we assume that the thermal flows $\nabla \theta . n$ equals 0 . In a radiative point of view, we model the air exit as a black body at a temperature $\theta_{\text {exit }}$ given (i.e. we assume that the exit absorbs all the radiant energy and emits at the temperature $\left.\theta_{\text {exit }}\right)$.

We denote by $\gamma_{d}^{\theta}$ the boundary part where the temperature is known (Dirichlet's condition) and by $\gamma_{n}^{\theta}$ the boundary part where the heat exchange is zero (Neumann's condition). We have $\gamma_{d}^{\theta} \cup \gamma_{n}^{\theta} \cup \gamma_{f}^{\theta}=\partial \omega$ and meas $\left(\gamma_{d}^{\theta}\right)>0$.

We denote by $P e$ the Peclet number, $P e=\frac{\rho C_{p} U^{*} L^{*}}{\lambda}$, where $U^{*}$ is a characteristic velocity of the flow and $L^{*}$ is a characteristic length. We denote the Biot number $B i, B i=\frac{h L^{*}}{\lambda}\left(B i\right.$ is constant), and the dimensionless reals $\delta_{1}=\frac{L^{*} \sigma\left(\theta^{*}\right)^{3}}{\lambda}$ and $\delta_{2}=\frac{L^{*} w^{*}}{\lambda \theta^{*}}$, where $\theta^{*}$ is a characteristic temperature and $w^{*}$ is a characteristic radiative energy. The dimensionless model is:

$\left(P^{\psi}\right)$ Find $\psi$ which satisfies

$$
\begin{array}{rll}
-\Delta \psi & =0 & \text { in } \omega \\
\psi & =\psi_{d} & \text { on } \gamma_{d}^{\psi} \\
\frac{\partial \psi}{\partial n} & =\psi_{n} & \text { on } \gamma_{n}^{\psi}
\end{array}
$$




$$
\begin{array}{rlrl}
\left(P^{\theta}\right) \text { Let } \vec{u}=\vec{\nabla} \psi, \text { find } & (\theta, w) \text { which satisfies } & \\
-\frac{1}{P e} \Delta \theta+\vec{u} \cdot \vec{\nabla} \theta & = & 0 & \text { in } \omega \\
\theta & = & \theta_{d} & \text { on } \gamma_{d}^{\theta} \\
\frac{\partial \theta}{\partial n} & = & 0 & \text { on } \gamma_{n}^{\theta} \\
-\frac{\partial \theta}{\partial n} & = & B i\left(\theta-\theta_{0}\right)+\frac{\varepsilon}{(1-\varepsilon)}\left(\delta_{1} \theta^{4}-\delta_{2} w\right) & \text { on } \gamma_{f}^{\theta} \\
w(x) & = & (1-\varepsilon(x)) \int_{\partial \omega} \phi(x, y) w(y) d s(y) & \\
& & +\varepsilon(x) \frac{\delta_{1}}{\delta_{2}} \theta^{4}(x) & \text { on } \partial \omega
\end{array}
$$

The functions $\psi_{d}$ and $\psi_{n}$ are respectively given in $H^{\frac{3}{2}}\left(\gamma_{d}^{\psi}\right)$ and $H^{\frac{1}{2}}\left(\gamma_{n}^{\psi}\right)$. The temperatures $\theta_{d}$ and $\theta_{0}$ are given positive functions respectively in $H^{\frac{3}{2}}\left(\gamma_{d}^{\theta}\right)$ and $H^{\frac{1}{2}}\left(\gamma_{f}^{\theta}\right) \cap L^{\infty}\left(\gamma_{f}^{\theta}\right)$

We make below an assumption on the domain $\omega$ in order to prove later some extra regularity on the solutions. As a matter of fact, if the fluid velocity $\vec{u}$ is regular enough, we can well define the coupling between the fluid model and the thermal model in standard functional spaces (see Theorem 3.1). In addition, this extra regularity on the fluid velocity and on the temperature is useful in order to establish in the numerical analysis the rate of convergence of the finite element schemes (see Section 5).

Assumption 3.1 Let $\omega$ be the domain of the equations. Then, it is a polygon of $\mathbb{I}^{2}$, it has re-entrant corners of maximal value $(2 \pi-\varepsilon)$ radians, for a given positive $\epsilon$ ( $\omega$ has no crack). Moreover, if there exists angles such that the boundary conditions are not of the same type at each side of the vertex (e.g. Dirichlet-Neumann), then such angles are lower than $\pi$. 
Now, we make an assumption on the compatibility between the boundary conditions of the fluid model and the boundary conditions of the thermal model in order to have the coupled problem well posed.

Assumption 3.2 We assume that the boundary conditions are such that $\vec{u} \cdot \vec{n}$ is given and is positive on $\gamma_{n}^{\theta} \cup \gamma_{f}^{\theta}$.

This assumption 3.2 expresses that where the temperature is not imposed, the fluid is outgoing. Let us notice that this assumptions 3.1 and 3.2 are satisfied in our case (Fig. 4). We define:

$$
\theta_{i n f}=\operatorname{Min}\left(\inf _{\gamma_{d}^{\theta}} \theta_{d}, \inf _{\gamma_{f}^{\theta}} \theta_{0}\right) \text { and } \theta_{s u p}=\operatorname{Max}\left(\sup _{\gamma_{d}^{\theta}} \theta_{d}, \sup _{\gamma_{f}^{\theta}} \theta_{0}\right)
$$

and

$$
\lambda_{\min }=4\left(C_{\Omega}^{2}+1\right) \varepsilon_{1} \sigma \theta_{\text {sup }}^{3}\left(\theta^{*}\right)^{3} L^{*}
$$

where $C_{\Omega}$ is the Poincare constant.

The thermal model $\left(P^{\theta}\right)$ with $\vec{u}$ given, has been studied in details in Ref. 1; and we recall below a result of existence and uniqueness of its solution.

Theorem 3.1 (Monnier and Vila, Ref. 1). Under Assumption 3.2, if the fluid is incompressible $(\operatorname{div}(\vec{u})=0$ in $\omega)$, if there exists a real $p>n$ such that $\vec{u} \in\left(L^{p}(\omega)\right)^{n}$, $n$ being the dimensional space, and if the thermal conductivity $\lambda$ is strictly greater than $\lambda_{\min }$ then there exists a unique solution $(\theta, w) \in H^{1}(\Omega) \times L^{\infty}(\partial \Omega)$ to Problem $\left(P^{\theta}\right)$ which satisfies

$$
\theta_{\text {inf }} \leq \theta \leq \theta_{\text {sup }} \text { in } \bar{\omega}
$$


Using Theorem 3.1 and by proving some extra regularity on the solutions, we obtain the main result of this section:

Theorem 3.2 Under the assumptions 3.1 and 3.2 and if the thermal conductivity $\lambda$ is strictly greater than $\lambda_{\min }$ then the coupled problem $\left(P^{\psi}\right)-\left(P^{\theta}\right)$ has a unique weak solution $(\psi, \theta, w) \in H^{1}(\omega) \times H^{1}(\omega) \times L^{\infty}(\partial \omega)$ which satisfies (20).

Furthermore, $\vec{u}=\vec{\nabla} \psi$ belongs to $H^{\alpha}(\omega)$ with $\alpha=\frac{1}{2}-\varepsilon$ for any $\varepsilon>0$, and $\theta$ belongs to $H^{1+\beta}(\omega)$ with $\beta=\frac{1}{2}-\varepsilon$ for any $\varepsilon>0$.

Proof. It follows from the Lax-Milgram theorem that there exists a unique $\psi \in$ $H^{1}(\omega)$ solution of $\left(P^{\psi}\right)$. Hence, there exists a unique velocity $\vec{u}$ in $\left(L^{2}(\omega)\right)^{2}$. We prove the existence and uniqueness of the solution of Problem $\left(P^{\theta}\right)$ using Theorem 3.1. By this end, we need to prove that $\vec{u} \in\left(L^{p}(\omega)\right)^{2}$ with $p>2$, which we do now. It follows from Assumption 3.1 and Grisvard's results, Ref. 14, that the unique solution $\psi$ of $\left(P^{\psi}\right)$ belongs to $H^{1+\alpha}(\omega)$ with $\alpha=\frac{1}{2}-\varepsilon$, for all $\varepsilon>0$. Thus, $\vec{u} \in\left(H^{\alpha}(\omega)\right)^{2}$ and $H^{\alpha}(\omega) \subset L^{p}(\omega)$ for all $p<4-\varepsilon^{\prime}$ where $\varepsilon^{\prime}$ vanishes with $\varepsilon$. Therefore, the regularity required on the fluid velocity is obtained and we can apply Theorem 3.1: if $\lambda>\lambda_{\min }$ then Problem $\left(P^{\theta}\right)$ has a unique weak solution $(\theta, w) \in H^{1}(\omega) \times L^{\infty}(\partial \omega)$ which satisfies $(20)$.

It remains us to prove some extra regularity on the temperature $\theta$. We do it using a bootstrap method applied to a new formulation of Problem $\left(P^{\theta}\right)$. It is proved in Refs. 15 and 11 that there exists a kernel $K(x, y)$ of $L^{1}(\partial \omega \times \partial \omega)$ which satisfies

$$
\int_{\partial \omega} K(x, y) \varepsilon(y) d s(y)=1-\varepsilon(x)
$$


and such that $\left(P^{\theta}\right)$ is equivalent to the following problem:

$\left(P^{Q}\right)$ Let $\vec{u}=\vec{\nabla} \psi$, find $\theta$ satisfying

$$
\begin{array}{rlll}
-\frac{1}{P e} \Delta \theta+u \cdot \nabla \theta & = & 0 & \text { in } \omega \\
\theta & = & \theta_{d} & \text { on } \gamma_{d}^{\theta} \\
\frac{\partial \theta}{\partial n} & = & 0 & \text { on } \gamma_{n}^{\theta} \\
-\frac{\partial \theta}{\partial n} & = & Q(\theta) & \text { on } \gamma_{f}^{\theta}
\end{array}
$$

with

$$
\begin{aligned}
Q(\theta)(x)= & B i\left(\theta-\theta_{0}\right)(x)+\left[\varepsilon(x) \delta_{1} \theta^{4}(x)\right. \\
& \left.-\frac{\varepsilon(x)}{1-\varepsilon(x)} \int_{\partial \omega} K(x, y) \varepsilon(y) \delta_{1} \theta^{4}(y) d s(y)\right] \text { a.e. on } \gamma_{f}^{\theta}
\end{aligned}
$$

Let us notice that this problem $\left(P^{Q}\right)$ has $\theta$ as only unknown. We refer to Ref. 15 (see also Ref. 11) for the expression of the kernel $K(x, y)$.

We use a bootstrap method on the problem $\left(P^{Q}\right)$ in order to prove some extra regularity on $\theta$. By this end, we consider the non principal part of the operator of $\left(P^{Q}\right)$ and boundary terms as data of the problem. We define the boundary term $g$ by $g=0$ on $\gamma_{n}^{\theta}, g=-Q(\theta)$ on $\gamma_{f}^{\theta}$. It follows from (20) and (21) that

$$
|Q(\theta)| \leq B_{i}\left(\theta_{\text {sup }}-\theta_{\text {inf }}\right)+\delta_{1}\left(\varepsilon_{1} \theta_{\text {sup }}^{4}-\varepsilon_{0} \theta_{\text {inf }}^{4}\right) \text { a.e. on } \partial \omega
$$

hence $g \in L^{\infty}\left(\gamma_{n}^{\theta} \cup \gamma_{f}^{\theta}\right)$. We define the right-hand side $f$ by $f=-P e u \nabla \theta$. We have $\theta \in H^{1}(\omega)$ and $u \in\left(H^{\frac{1}{2}-\varepsilon}(\omega)\right)^{2}$. In other respects, one has $H^{s}(\omega) \subset L^{q}(\omega)$ for $s \geq 0$ and $q \geq 2$ if $\frac{1}{q}>\frac{1}{2}-\frac{s}{2}$. Let $q^{\prime}$ be such that $\frac{1}{q}+\frac{1}{q^{\prime}}=1$, then $L^{q^{\prime}}(\omega) \subset H^{-s}(\omega)$ if $1-\frac{1}{q^{\prime}}>\frac{1}{2}-\frac{s}{2}$ i.e. if $s>\frac{2}{q^{\prime}}-1$. Then, it follows that $f \in L^{q^{\prime}}(\omega)$ for all $q^{\prime}<\frac{4}{3}$, hence $f \in H^{-\frac{1}{2}-\varepsilon}(\omega)$ for all $\varepsilon>0$.

Finally, the solution of $\left(P^{Q}\right)$ satisfies:

$$
\left\{\begin{array}{ccl}
-\Delta \theta & =f & \text { in } \omega \\
\theta & =\theta_{d} & \text { on } \gamma_{d}^{\theta} \\
\frac{\partial \theta}{\partial n} & =g & \text { on } \gamma_{n}^{\theta} \cup \gamma_{f}^{\theta}
\end{array}\right.
$$


We deduce from Assumption 3.1 and results of regularity stated in Ref. 14 that the solution $\theta$ belongs to $H^{1+\beta}(\omega)$ with $\beta=\frac{1}{2}-\varepsilon$ for all $\varepsilon>0$. This completes the proof.

Remark 3.1 In the bidimensional case and under Assumption 3.1, it follows from Ref. 14 that $u \in\left(L^{p}(\omega)\right)^{2}$ with $p>2$. On the other hand, in the three dimensional case and if we suppose the same regularity as above, $u$ does not belong to $\left(L^{p}(\omega)\right)^{3}$ with $p>3$ and the regularity required in order to obtain existence of a solution to Problem $\left(P^{\theta}\right)$ is not satisfied. Thus, the coupled system (potential flow - thermal with convective term) $\left(P^{\psi}\right)-\left(P^{\theta}\right)$ is not defined in three dimensions of space when the domain has a re-entrant corner of $(2 \pi-\varepsilon)$ radians. But, if $\psi \in H^{2}(\omega)$ then $u \in\left(H^{1}(\omega)\right)^{3}$ and $u \in\left(L^{p}(\omega)\right)^{3}$ with $p>3$ and the regularity required is obtained. Nevertheless, in order to model an air flow under a car hood, the "right" fluid model are the steady incompressible Navier-Stokes equations. And, the velocity $u$ is more regular than in the present model and the required regularity conditions are obtained.

\section{Analysis of the Optimal Design Problem}

In this section, we write the continuous equations which permit to treat the shape optimal design problem as applied to the cooling problem under a car hood. In others words, we apply the technic of Section 2.2.3 (more specifically Corollary 2.1 and Proposition 2.2) when the state equation (10) is the weak formulation of the problem $\left(P^{\psi}\right)-\left(P^{\theta}\right)$ studied in Section 3. Let us notice that in the present section, we do not precise in concrete terms what are the design variables, yet. We do it 
later in Section 5.3. The main goal of the present section is the following: i) to make fit the model $\left(P^{\psi}\right)-\left(P^{\theta}\right)$ into the framework of Corollary 2.1; ii) to prove that the assumptions of this corollary are satisfied (especially that the solution is differentiable with respect to the domain); iii) then, to give an exact expression of the adjoint state equation and an exact expression of the differential of the cost function. These results are presented in Proposition 4.1.

\subsection{Position of the Problem}

Let $\hat{\Omega}$ be a lipschitz bounded open set in $\mathbb{R}^{2}$, and let $\hat{T}_{0} \in \hat{\mathcal{T}}^{1}$ (see section 2.2). We denote $\Omega=\hat{T}_{0}(\hat{\Omega})$ and we consider an open set $\omega=T(\Omega)$ where $T \in \mathcal{T}^{1}$ (or equivalently $\omega \in \mathcal{D}$ ) such that Assumption 3.1 remains true.

State Equation We define the following vectorial spaces and affine sub-spaces:

$$
\begin{gathered}
V_{0}^{\psi}(\omega)=\left\{\psi \in H^{1}(\omega) ;\left.\psi\right|_{\gamma_{d}^{\psi}}=0\right\} ; \quad V_{t}^{\psi}(\omega)=\left\{\psi \in H^{1}(\omega) ;\left.\psi\right|_{\gamma_{d}^{\psi}}=\psi_{d}\right\} \\
V_{0}^{\theta}(\omega)=\left\{\theta \in H^{1}(\omega) ;\left.\theta\right|_{\gamma_{d}^{\theta}}=0\right\} ; \quad V_{t}^{\theta}(\omega)=\left\{\theta \in H^{1}(\omega) ;\left.\theta\right|_{\gamma_{d}^{\theta}}=\theta_{d}\right\} \\
V_{t}(\omega)=V_{t}^{\psi}(\omega) \times V_{t}^{\theta}(\omega) \times L^{2}(\partial \omega) ; \quad V_{0}(\omega)=V_{0}^{\psi}(\omega) \times V_{0}^{\theta}(\omega) \times L^{2}(\partial \omega)
\end{gathered}
$$

The state equation is:

Find $y^{\omega}=\left(\psi^{\omega}, \theta^{\omega}, w^{\omega}\right) \in V_{t}(\omega)$ such that

$$
\forall z=(\varphi, t, v) \in V_{0}(\omega), E_{\omega}\left(y^{\omega}, z\right)=0
$$

where $E_{\omega}(y, z)$ is the sum of the variational formulations of the fluid partial differential equation, the thermal equation and the integral equation. Its expression 
is:

$$
\begin{aligned}
E_{\omega}(y, z) & =\int_{\omega}\langle\nabla \psi, \nabla \varphi\rangle d x-\int_{\gamma_{n}^{\nu}} \psi_{n} \varphi d s \\
& +\int_{\omega}<\nabla \theta, \nabla t>d x+P e \int_{\omega}<u, \nabla \theta>t d x+\int_{\gamma_{f}^{\theta}} B i\left(\theta-\theta_{0}\right) t d s \\
& +\int_{\gamma_{f}^{\theta}} \frac{\varepsilon}{(1-\varepsilon)}\left(\delta_{1} \theta^{4}-\delta_{2} w\right) t d s \\
& +\int_{\partial \omega} w v d s-\int_{\partial \omega}(1-\varepsilon(x))\left[\int_{\partial \omega} \phi(x, y) w(y) d s(y)\right] v(x) d s(x) \\
& -\frac{\delta_{1}}{\delta_{2}} \int_{\partial \omega} \varepsilon \theta^{4} v d s
\end{aligned}
$$

This state equation is equivalent to a system of two partial differential equations and an integral equation as follows:

Find $\psi^{\omega} \in V_{t}^{\psi}(\omega)$ such that

$$
\forall \varphi \in V_{0}^{\psi}(\omega), \int_{\omega}\left\langle\nabla \psi^{\omega}, \nabla \varphi>d x=\int_{\gamma_{n}^{\psi}} \psi_{n} \varphi d s\right.
$$

Let $u^{\omega}=\nabla \psi^{\omega}$, find $\left(\theta^{\omega}, w^{\omega}\right) \in V_{t}^{\theta}(\omega) \times L^{2}(\partial \omega)$ such that

$$
\begin{gathered}
\left.\forall t \in V_{0}^{\theta}(\omega), \quad \int_{\omega}<\nabla \theta^{\omega}, \nabla t>d x+P e \int_{\omega}<u^{\omega}, \nabla \theta^{\omega}\right\rangle t d x \\
+\int_{\gamma_{f}^{\theta}} B i\left(\theta^{\omega}-\theta_{0}\right) t d s+\int_{\gamma_{f}^{\theta}} \frac{\varepsilon}{(1-\varepsilon)}\left(\delta_{1}\left(\theta^{\omega}\right)^{4}-\delta_{2} w^{\omega}\right) t d s=0 \\
\forall v \in L^{2}(\partial \omega), \quad \int_{\partial \omega} w^{\omega} v d s-\int_{\partial \omega}(1-\varepsilon(x))\left[\int_{\partial \omega} \phi(x, y) w^{\omega}(y) d s(y)\right] v(x) d s(x) \\
=\frac{\delta_{1}}{\delta_{2}} \int_{\partial \omega} \varepsilon\left(\theta^{\omega}\right)^{4} v d s
\end{gathered}
$$

Under the assumptions of Theorem 3.2, there exists a unique solution $y^{\omega} \in V_{t}(\omega)$ to the state equation (23) which satisfies the weak maximum principle (20).

Control Problem We seek to optimize the hose shape (see Figure 4) in order to minimize the temperature on its boundary. More precisely, we seek to minimize the $L^{2}$-norm of the temperature on the hose boundary. We denote by $\gamma_{\text {hose }}$ the hose boundary, $\left(\gamma_{\text {hose }}\right.$ is a part of $\left.\gamma_{f}^{\theta}\right)$, and we consider the following observation function:

$$
J_{\omega}: V_{t}^{\theta}(\omega) \rightarrow \mathbb{R}: \theta \mapsto \frac{1}{2} \int_{\gamma_{\text {hose }}} \theta^{2} d s
$$


Then, we define the cost function as follows:

$$
j: \mathcal{D} \rightarrow \mathbb{R} ; \omega \mapsto j(\omega)=J_{\omega}\left(y^{\omega}\right)
$$

where $y^{\omega}$ is the solution of the state equation (23). (The cost function depends on $\omega$ through the boundary $\gamma_{\text {hose }}$ and through the temperature $\left.\theta^{\omega}\right)$. The minimization problem is:

Find $\omega^{\star} \in \mathcal{D}$ such that

$$
j\left(\omega^{\star}\right)=\min _{\omega \in \mathcal{D}} j(\omega)
$$

We will detail in Section 5.3 what are numerically, the design variables.

\subsection{Differential of the cost function}

In this section, we apply Corollary 2.1 to the optimal design problem presented above. Following Section 2.2, we transport the state equation (23) to the reference domain $\Omega$. It gives:

Find $\bar{y}^{T}=\left(\bar{\psi}^{T}, \bar{\theta}^{T}, \bar{w}^{T}\right) \in V_{t}(\Omega)$ such that

$$
\forall \bar{z}=(\bar{\varphi}, \bar{t}, \bar{v}) \in V_{0}(\Omega), \bar{E}\left(T ; \bar{y}^{T}, \bar{z}\right)=0
$$

We recall that for $T \in \mathcal{T}^{1}$, the mapping $z \in V_{0}(T(\Omega)) \mapsto z \circ T \in V_{0}(\Omega)$ is an isomorphism so that $\bar{y}^{T}=y^{\omega} \circ T \in V_{t}(\Omega)$ exists and is unique.

We write:

$$
\bar{\jmath}(T)=j(\omega)=\bar{J}\left(T ; \bar{y}^{T}\right)=\frac{1}{2} \int_{\Gamma_{\text {hose }}}\left(\bar{\theta}^{T}\right)^{2}|\operatorname{det} D T|\left\|{ }^{t} D T^{-1} \cdot n\right\|_{\mathbb{R}^{2}} d \bar{s}
$$

It is clear that the functional $\bar{J}: \mathcal{T}^{1} \times V_{0}(\Omega) \rightarrow \mathbb{R}:(T, \bar{y}) \mapsto \bar{J}(T, \bar{y})$ is continuously differentiable with respect to $(T ; \bar{y})$.

Proposition 4.1 If the thermal conductivity $\lambda$ is strictly greater than $\lambda_{\min }$ (see (19)) then there exists $\mathcal{V}(T)$, a neighborhood of $T$, such that the cost function $j$ : 
$\omega \in \mathcal{D} \mapsto j(\omega) \in \mathbb{R}$ is continuously differentiable for all $\omega=T(\Omega), T \in \mathcal{V}(T) \subset \mathcal{T}^{1}$.

Also, for all $V \in \operatorname{Lip}\left(\Omega, \mathbb{R}^{2}\right)$, we have

$$
\frac{d j}{d \omega}(\Omega) \cdot V=\frac{\partial J_{\Omega}}{\partial \omega}\left(y^{\Omega}\right) \cdot V-\frac{\partial E_{\Omega}}{\partial \omega}\left(y^{\Omega}, p^{\Omega}\right) \cdot V
$$

where $y^{\Omega}$ is the solution of the state equation (23) posed in $\Omega$ and $p^{\Omega}$ is the unique solution of the adjoint state equation:

Find $p^{\Omega}=\left(P^{\Omega}, T^{\Omega}, q^{\Omega}\right) \in V_{0}(\Omega)$ such that

$$
\forall z=(\varphi, t, v) \in V_{0}(\Omega), \frac{\partial E_{\Omega}}{\partial y}\left(y^{\Omega}, p^{\Omega}\right) \cdot z=\frac{\partial J_{\Omega}}{\partial y}\left(y^{\Omega}\right) \cdot z
$$

Moreover (26) can be expressed by:

$$
\frac{\partial J_{\Omega}}{\partial \omega}\left(y^{\Omega}\right) \cdot V=\frac{1}{2} \int_{\Gamma_{\text {hose }}}\left(\theta^{\Omega}\right)^{2}<\tau, D V \tau>d s
$$

where $\tau$ is the unit tangent vector to $\partial \Omega$ and $<., .>$ denotes the inner product in $\mathbb{R}^{2}$.

$\frac{\partial E_{\Omega}}{\partial \omega}\left(y^{\Omega}, p^{\Omega}\right) \cdot V=\frac{\partial E_{\Omega}^{\psi}}{\partial \omega}\left(y^{\Omega}, p^{\Omega}\right) \cdot V+\frac{\partial E_{\Omega}^{\theta}}{\partial \omega}\left(y^{\Omega}, p^{\Omega}\right) \cdot V+\frac{\partial E_{\Omega}^{w}}{\partial \omega}\left(y^{\Omega}, p^{\Omega}\right) \cdot V$

with

$$
\begin{aligned}
\frac{\partial E_{\Omega}^{\psi}}{\partial \omega}\left(y^{\Omega}, p^{\Omega}\right) \cdot V & =\int_{\Omega}<\nabla \psi^{\Omega}, \nabla P^{\Omega}>\operatorname{div} V d x \\
& -\int_{\Omega}<\left(D V+{ }^{T} D V\right) \nabla \psi^{\Omega}, \nabla P^{\Omega}>d x \\
& -\int_{\Gamma_{n}^{\psi}} \psi_{n} P^{\Omega}<\tau, D V \tau>d s
\end{aligned}
$$

(with $u^{\Omega}=\nabla \psi^{\Omega}$ ),

$$
\begin{aligned}
\frac{\partial E_{\Omega}^{\theta}}{\partial \omega}\left(y^{\Omega}, p^{\Omega}\right) \cdot V & =\int_{\Omega}<\nabla \theta^{\Omega}, \nabla T^{\Omega}>\operatorname{div} V d x \\
& +P e \int_{\Omega}<u^{\Omega}, \nabla \theta^{\Omega}>T^{\Omega} \operatorname{div} V d x
\end{aligned}
$$




$$
\begin{aligned}
& -\int_{\Omega}<\left(D V+{ }^{T} D V\right) \nabla \theta^{\Omega}, \nabla T^{\Omega}>d x \\
& -\operatorname{Pe} \int_{\Omega}<D V u^{\Omega}, \nabla \theta^{\Omega}>T^{\Omega} d x \\
& +\int_{\Gamma_{f}^{\theta}}\left[B i\left(\theta^{\Omega}-\theta_{0}\right)+\frac{\varepsilon}{(1-\varepsilon)}\left(\delta_{1}\left(\theta^{\Omega}\right)^{4}-\delta_{2} w^{\Omega}\right)\right] T^{\Omega}<\tau, D V \tau>d s
\end{aligned}
$$

$$
\begin{aligned}
\frac{\partial E_{\Omega}^{\omega}}{\partial \omega}\left(y^{\Omega}, p^{\Omega}\right) \cdot V & =\int_{\partial \Omega}\left(w^{\Omega}-\varepsilon \frac{\delta_{1}}{\delta_{2}}\left(\theta^{\Omega}\right)^{4}\right) q^{\Omega}<\tau, D V \tau>d s \\
& -\int_{\partial \Omega}(1-\varepsilon(x))\left[\int_{\partial \Omega} \phi(x, y) w^{\Omega}(y)(<\tau, D V \tau>(y)\right. \\
& +<\tau, D V \tau>(x)) d s(y)] q^{\Omega}(x) d s(x)
\end{aligned}
$$

Proof. We write the state equation (25) as follows:

$$
\bar{y}^{T} \in V_{t}(\Omega): \overline{\mathcal{E}}\left(T ; \bar{y}^{T}\right)=0 \text { in } V_{0}^{\prime}(\Omega)
$$

where the operator $\overline{\mathcal{E}}$ is defined by $<\overline{\mathcal{E}}\left(T ; \bar{y}^{T}\right), \bar{z}>_{V_{0}^{\prime} \times V_{0}}=\bar{E}\left(T ; \bar{y}^{T}, \bar{z}\right)$ for all $\bar{z} \in$ $V_{0}(\Omega)$. It is easy to prove that the mapping $\bar{E}(T ; \bar{y},$.$) is continuously differentiable$ with respect to $(T, \bar{y})$ from $\mathcal{T}^{1} \times V_{0}(\Omega)$ onto $\mathcal{L}\left(V_{0}(\Omega) ; I R\right)$ which means that $\overline{\mathcal{E}}$ belongs to $C^{1}\left(\mathcal{T}^{1} \times V_{0}(\Omega) ;\left(V_{0}(\Omega)\right)^{\prime}\right)$

In order to apply Corollary 2.1, we still have to prove that the linearized problem is well posed. Using the Lax-Milgram theorem, it is proved in (Ref. 1, Proposition 4) that if the thermal conductivity $\lambda$ is greater than $\lambda_{\min }$, the linearized thermal model is well posed. Hence, under this condition, the linearized problem is well posed and the result follows from Corollary 2.1 and Proposition 2.2.

The adjoint state equation (27) is equivalent to a system of two linear partial differential equations and an integral equation. These equations are solved in the 
reverse way compared to the state equation and each of them is the adjoint equation of the corresponding equation to the direct problem. They are:

Find $\left(T^{\Omega}, q^{\Omega}\right) \in V_{0}^{\theta}(\Omega) \times L^{2}(\partial \Omega)$ such that

$$
\begin{aligned}
& \forall v \in L^{2}(\partial \Omega), \quad \int_{\partial \Omega} q^{\Omega} v d s-\int_{\partial \Omega}(1-\varepsilon(x))\left[\int_{\partial \Omega} \phi(x, y) v(y) d s(y)\right] q^{\Omega}(x) d s(x) \\
& =\delta_{2} \int_{\Gamma_{f}^{\theta}} \frac{\varepsilon}{(1-\varepsilon)} T^{\Omega} v d s \\
& \forall t \in V_{0}^{\theta}(\Omega), \int_{\Omega}<\nabla T^{\Omega}, \nabla t>d x \\
& +P e \int_{\Omega}<u^{\Omega}, \nabla t>T^{\Omega} d x+\int_{\Gamma_{f}^{\theta}}\left[B i+4 \delta_{1} \frac{\varepsilon}{(1-\varepsilon)}\left(\theta^{\Omega}\right)^{3}\right] T^{\Omega} t d s \\
& =4 \frac{\delta_{1}}{\delta_{2}} \int_{\Gamma_{f}^{\theta}} \varepsilon\left(\theta^{\Omega}\right)^{3} q^{\Omega} t d s+\int_{\Gamma_{\text {hose }}} \theta^{\Omega} t d s
\end{aligned}
$$

Let $T^{\Omega}$, find $P^{\Omega} \in V_{0}^{\psi}(\Omega)$ such that

$$
\forall \varphi \in V_{0}^{\psi}(\Omega), \int_{\Omega}<\nabla P^{\Omega}, \nabla \varphi>d x=-P e \int_{\Omega} T^{\Omega}<\nabla \theta^{\Omega}, \nabla \varphi>d x
$$

\section{Numerical Analysis}

First, we discretize the state and adjoint state equations using a finite element method. We present some results of existence, uniqueness of the discrete solutions and convergence towards the exact solutions (theorems 5.1 and 5.2). Then, we discretize the expression (26) of the differential of the cost function in order to obtain the shape gradient (see the expression (33)). Finally, we detail in Section 5.3 the implementation of the problem. By this end, we describe the algorithms we use in order to solve the state equation. Then, we detail how we define numerically the hose shape, in others words what are the design variables. We present a technic to compute the vector field $\vec{V}$ of the perturbation of domain and finally we summarize the optimization process in Figure 5. 


\subsection{Discrete State Equations and Adjoint State Equations}

We discretize the state and adjoint state equations using a finite element method.

We denote by $\left(\mathcal{T}_{h}\right)$ a regular and quasi-uniform family of triangulation, $\bar{\Omega}=\cup_{\left(T \in \mathcal{T}_{h}\right)} T$.

We associate this family of triangulation to a $\mathcal{C}^{0}$ Lagrange reference finite element.

Let $k$ be an integer greater or equal to 1 . We denote by $P_{k}$ the set of polynomials of degree lower or equal to $k$. We define the following discrete spaces:

$$
\begin{gathered}
X_{h}(\Omega)=\left\{x_{h} \in \mathcal{C}^{0}(\bar{\Omega}) ; \forall T \in \mathcal{T}_{h},\left.x_{h}\right|_{T} \in P_{k}\right\} \\
V_{0 h}^{\psi}(\Omega)=\left\{\varphi_{h} \in X_{h}(\Omega) ;\left.\varphi_{h}\right|_{\Gamma_{d}^{\psi}}=0\right\} \\
V_{0 h}^{\theta}(\Omega)=\left\{t_{h} \in X_{h}(\Omega) ;\left.t_{h}\right|_{\Gamma_{d}^{\theta}}=0\right\}
\end{gathered}
$$

We assume that $\psi_{d}$ and $\theta_{d}$ belong to $X_{h}(\Omega)$ and we denote respectively by $V_{t h}^{\psi}(\Omega)$ and $V_{t h}^{\theta}(\Omega)$ the affine sub-spaces of $V_{0 h}^{\psi}(\Omega)$ and $V_{0 h}^{\theta}(\Omega)$; namely they are such that $\left.\varphi_{h}\right|_{\Gamma_{d}^{\psi}}=\psi_{d}$ and $\left.t_{h}\right|_{\Gamma_{d}^{\theta}}=\theta_{d}$, respectively. We define

$$
\mathcal{W}_{h}(\partial \Omega)= \begin{cases}\{v: \partial \Omega \rightarrow \mathbb{R}, v \text { piecewise constant }\} & \text { if } k=1 \\ \left\{v \in \mathcal{C}^{0}(\partial \Omega) ;\left.v\right|_{\partial T} \in P_{k-1} \forall \partial T \in \partial \mathcal{T}_{h}\right\} & \text { if } k \geq 2\end{cases}
$$

where $\partial \mathcal{T}_{h}=\left\{\partial T \cap \partial \Omega: \partial T \cap \partial \Omega \neq \emptyset, T \in \mathcal{T}_{h}\right\}$. All these spaces and affine sub-spaces are respectively subsets of $H^{1}(\Omega)$ and $L^{2}(\partial \Omega)$.

State Equation The discrete formulation of the state equation is: $\left(P_{h}^{\psi}\right)$ Find $\psi_{h}^{\Omega} \in V_{t h}^{\psi}(\Omega)$ such that

$$
\begin{aligned}
& \forall \varphi_{h} \in V_{0 h}^{\psi}(\Omega), \quad \int_{\Omega}<\nabla \psi_{h}^{\Omega}, \nabla \varphi_{h}>d x=\int_{\Gamma_{n}^{\psi}} \psi_{n} \varphi_{h} d s \\
& \left(P_{h}^{\theta}\right) \text { Let } u_{h}^{\Omega}=\nabla \psi_{h}^{\Omega} \text {, find }\left(\theta_{h}^{\Omega}, w_{h}^{\Omega}\right) \in V_{t h}^{\theta}(\Omega) \times \mathcal{W}_{h}(\partial \Omega) \text { such that } \\
& \forall t_{h} \in V_{0 h}^{\theta}(\Omega), \quad \int_{\Omega}<\nabla \theta_{h}^{\Omega}, \nabla t_{h}>d x+\operatorname{Pe} \int_{\Omega}<u_{h}^{\Omega}, \nabla \theta_{h}^{\Omega}>t_{h} d x \\
& +\int_{\Gamma_{f}^{\theta}}\left[B i\left(\theta_{h}^{\Omega}-\theta_{0}\right)+\frac{\varepsilon}{(1-\varepsilon)}\left(\delta_{1}\left(\theta_{h}^{\Omega}\right)^{4}-\delta_{2} w_{h}^{\Omega}\right)\right] t_{h} d s=0 \\
& \forall v_{h} \in \mathcal{W}_{h}(\partial \Omega), \quad \int_{\partial \Omega}(I-A) w_{h}^{\Omega} v_{h} d s=\frac{\delta_{1}}{\delta_{2}} \int_{\partial \Omega} \varepsilon\left(\theta_{h}^{\Omega}\right)^{4} v_{h} d s
\end{aligned}
$$


where $A$ is the operator defined from $L^{2}(\partial \Omega)$ into $L^{2}(\partial \Omega)$ by:

$$
A w(x)=(1-\varepsilon(x)) \int_{\partial \Omega} \phi(x, y) w(y) d s(y)
$$

We present below the results of existence and uniqueness of the discrete solutions and their convergence towards the exact solutions.

Theorem 5.1 There exists a unique solution $\psi_{h}^{\Omega} \in V_{t h}^{\psi}(\Omega)$ to $\left(P_{h}^{\psi}\right)$. Furthermore,

$$
\left\|u^{\Omega}-u_{h}^{\Omega}\right\|_{0, \Omega}=O\left(h^{\alpha}\right)
$$

where $\alpha$ is a strictly positive number such that $\psi^{\Omega}$, the unique solution of $\left(P^{\psi}\right)$, belongs to $H^{1+\alpha}(\Omega)$, and $O\left(h^{\alpha}\right)$ is a function such that $\frac{O\left(h^{\alpha}\right)}{h^{\alpha}}$ is bounded when the mesh size $h$ tends to 0 .

Proof. The existence and uniqueness follows from the Lax-Milgram theorem, the regularity of $\psi^{\Omega}$ was discussed in Theorem 3.2 and the convergence result follows from approximation properties in fractional Sobolev spaces (see Refs. 1 and 11 for a complete proof).

The numerical analysis of a thermal model slightly different from $\left(P_{h}^{\theta}\right)$ is written in details in Ref. 1. Let us recall the result established in Ref. 1 . We consider $\varphi(t)$ an operator of truncation of class $C^{2}$ defined as follows:

$$
\varphi(t)=\left\{\begin{array}{ccc}
\theta_{\text {inf }} & \text { if } & t \leq \theta_{\text {inf }}-\xi \\
P_{\text {inf }}(t) & \text { if } & \theta_{\text {inf }}-\xi \leq t \leq \theta_{\text {inf }} \\
t & \text { if } & \theta_{\text {inf }} \leq t \leq \theta_{\text {sup }} \\
P_{\text {sup }}(t) & \text { if } & \theta_{\text {sup }} \leq t \leq \theta_{\text {sup }}+\xi \\
\theta_{\text {sup }} & \text { if } & \theta_{\text {sup }}+\xi \leq t(x)
\end{array}\right.
$$


where $\xi$ is a small real such that $\theta_{\text {inf }}>\xi>0$ and the polynomial $P_{\text {inf }}(t)$ and $P_{\text {sup }}(t)$ are such that $\varphi(t)$ is $C^{2}$. We define the truncated model denoted $\left(\bar{P}_{h}^{\theta}\right)$ :

$$
\begin{aligned}
& \left(\bar{P}_{h}^{\theta}\right) \text { Let } u_{h}^{\Omega}=\nabla \psi_{h}^{\Omega} \text {, find }\left(\theta_{h}^{\Omega}, w_{h}^{\Omega}\right) \in V_{t h}^{\theta}(\Omega) \times \mathcal{W}_{h}(\partial \Omega) \text { such that } \\
& \forall t_{h} \in V_{0 h}^{\theta}(\Omega), \quad \int_{\Omega}<\nabla \theta_{h}^{\Omega}, \nabla t_{h}>d x+P e \int_{\Omega}<u_{h}^{\Omega}, \nabla \theta_{h}^{\Omega}>t_{h} d x \\
& +\int_{\Gamma_{f}^{\theta}}\left[B i\left(\varphi\left(\theta_{h}^{\Omega}\right)-\theta_{0}\right)+\frac{\varepsilon}{(1-\varepsilon)}\left(\delta_{1} \varphi\left(\theta_{h}^{\Omega}\right)^{4}-\delta_{2} w_{h}^{\Omega}\right)\right] t_{h} d s=0 \\
& \forall v_{h} \in \mathcal{W}_{h}(\partial \Omega), \quad \int_{\partial \Omega}(I-A) w_{h}^{\Omega} v_{h} d s=\frac{\delta_{1}}{\delta_{2}} \int_{\partial \Omega} \varepsilon \varphi\left(\theta_{h}^{\Omega}\right)^{4} v_{h} d s
\end{aligned}
$$

We assume that:

$$
\inf _{\Gamma_{d}^{\theta}} \theta_{d}<\inf _{\Gamma_{f}^{\theta}} \theta_{0} \text { and } \sup _{\Gamma_{f}^{\theta}} \theta_{0}<\sup _{\Gamma_{d}^{\theta}} \theta_{d}
$$

We assume that the emittance $\varepsilon(x)$ is regular enough (for example a lipschitz function) and we denote by $\gamma$ the greatest real (strictly positive) such that the radiosity $w^{\Omega}$, solution of $\left(P^{\theta}\right)$, belongs to $H^{\gamma}(\partial \Omega)$. The result proved in Ref. 1, Theorem 2, is the following:

Theorem 5.2 (Monnier-Vila, Ref. 1) Under the assumptions 3.1 and 3.2, if the thermal conductivity $\lambda$ is large enough and if the mesh size $h$ is small enough, then there exists a unique solution $\left(\theta_{h}^{\Omega}, w_{h}^{\Omega}\right)$ in $V_{t h}^{\theta}(\Omega) \times \mathcal{W}_{h}(\partial \Omega)$ to $\left(\bar{P}_{h}^{\theta}\right)$. Furthermore, there exists a constant $C$ independent of $h$ such that for all $\varepsilon>0$,

$$
\left\|\theta^{\Omega}-\theta_{h}^{\Omega}\right\|_{1, \Omega}+\left\|w^{\Omega}-w_{h}^{\Omega}\right\|_{0, \partial \Omega} \leq C h^{\alpha_{\varepsilon}}
$$

with $\alpha_{\varepsilon}=\operatorname{Min}(\alpha-\varepsilon, \beta-\varepsilon, \gamma)$, where $\psi^{\Omega} \in H^{1+\alpha}(\Omega),\left(\theta^{\Omega}, w^{\Omega}\right)$ is the unique solution of $\left(P^{\theta}\right), \theta^{\Omega} \in H^{1+\beta}(\Omega)$ and $w^{\Omega} \in H^{\gamma}(\partial \Omega)$. 
Let us notice that this numerical analysis has been written for the centered scheme i.e. without any stabilization procedure.

Adjoint State Equation The discrete formulation of the adjoint state equation is the following:

Let $u_{h}^{\Omega}$ and $\theta_{h}^{\Omega}$, find $\left(T_{h}^{\Omega}, q_{h}^{\Omega}\right) \in V_{0 h}^{\theta}(\Omega) \times \mathcal{W}_{h}(\partial \Omega)$ such that

$$
\begin{aligned}
& \forall v \in \mathcal{W}_{h}(\partial \Omega), \quad \int_{\partial \Omega} q_{h}^{\Omega} v_{h} d s-\int_{\partial \Omega}(1-\varepsilon(x))\left[\int_{\partial \Omega} \phi(x, y) v_{h}(y) d s(y)\right] q_{h}^{\Omega}(x) d s(x) \\
& \quad=\delta_{2} \int_{\Gamma_{f}^{\theta}} \frac{\varepsilon}{(1-\varepsilon)} T_{h}^{\Omega} v_{h} d s \\
& \forall t_{h} \in V_{0 h}^{\theta}(\Omega), \quad \int_{\Omega}<\nabla T_{h}^{\Omega}, \nabla t_{h}>d x+P e \int_{\Omega}<u_{h}^{\Omega}, \nabla t_{h}>T_{h}^{\Omega} d x \\
& +\int_{\Gamma_{f}^{\theta}}\left[B i+4 \delta_{1} \frac{\varepsilon}{(1-\varepsilon)}\left(\theta_{h}^{\Omega}\right)^{3}\right] T_{h}^{\Omega} t_{h} d s=4 \frac{\delta_{1}}{\delta_{2}} \int_{\Gamma_{f}^{\theta}} \varepsilon\left(\theta^{\Omega}\right)^{3} q_{h}^{\Omega} t_{h} d s+\int_{\Gamma_{h o s e}} \theta_{h}^{\Omega} t_{h} d s
\end{aligned}
$$

Let $T_{h}^{\Omega}$ and $\theta_{h}^{\Omega}$, find $P_{h}^{\Omega} \in V_{\mathrm{oh}}^{\psi}(\Omega)$ such that

$$
\forall \varphi_{h} \in V_{0 h}^{\psi}(\Omega), \int_{\Omega}<\nabla P_{h}^{\Omega}, \nabla \varphi_{h}>d x=-P e \int_{\Omega} T_{h}^{\Omega}<\nabla \theta_{h}^{\Omega}, \nabla \varphi_{h}>d x
$$

\subsection{Discretization of the Cost Gradient: Principle of the Method}

Let us recall that for a given $\Omega$, and a given vector field $V \in \operatorname{Lip}\left(\Omega ; \mathbb{R}^{2}\right)$, we have:

$$
\frac{d j}{d \omega}(\Omega) \cdot \vec{V}=\int_{\Omega} F\left(y^{\Omega}, p^{\Omega}\right) \cdot \vec{V} d x+\int_{\partial \Omega} f\left(y^{\Omega}, p^{\Omega}\right) \cdot \vec{V} d s
$$

where $y^{\Omega}$ and $p^{\Omega}$ are the solutions of the direct and adjoint state equations. Their expression is given in Proposition 4.1. We need to discretize this expression at two different levels:

- $V$ belongs to an infinite dimensional vector space which has to be approximated by a finite dimensional one, 
- $y^{\Omega}$ and $p^{\Omega}$ are solutions of partial differential equations, they have to be approximated.

\subsubsection{Approximation of $y^{\Omega}$ and $p^{\Omega}$}

Obviously, $y^{\Omega}$ and $p^{\Omega}$ will be approximated using the finite elements described in Section 5.1. We get $y_{h}^{\Omega}$ and $p_{h}^{\Omega}$, where $h$ is the mesh size, using the algorithms briefly detailed in Section 5.3. The discrete solutions $y_{h}^{\Omega}$ and $p_{h}^{\Omega}$ replace $y^{\Omega}$ and $p^{\Omega}$ in formula (31).

\subsubsection{Approximation of $\mathrm{V}$}

One must be careful because $V$ is defined on $\Omega$ which is moving in the algorithm.

Let us recall the definitions and properties which have been given in Section 2 (Definition 2.2 and Proposition 2.1):

$\hat{\Omega}$ is a reference open set

$\Omega=\hat{T}_{0}(\hat{\Omega}) \quad\left(\hat{T}_{0}\right.$ is a bilipschitzian homeomorphism)

$. V=\hat{V} \circ \hat{T}_{0}^{-1}(\hat{V}$ is defined on $\hat{\Omega}, V$ is defined on $\Omega)$

$\cdot \frac{d j}{d \omega}(\Omega) \cdot V=\frac{d \hat{j}}{d \hat{T}}\left(\hat{T}_{0}\right) \cdot \hat{V} \quad$ (Definition 2.2)

$$
=\frac{d \bar{y} \Omega}{d T}(I) \cdot V \quad(\text { Proposition 2.1) }
$$

This means that we have to choose a reference set $\hat{\Omega}$ and a finite dimensional space $\hat{\operatorname{Lip}}{ }_{H}$ approximating $\operatorname{Lip}\left(\hat{\Omega} ; \mathbb{R}^{2}\right)$. A vector $\hat{V}_{H}$ approximating $\hat{V}$ is chosen in $\hat{\operatorname{Lip}}{ }_{H}$. Notice that the mesh size $H$ is a priori different from the finite element mesh size $h$. In general, it would be prudent to choose $h$ smaller than $H$. As a matter of fact, $H$ gives the size of eventual oscillations of the boundary $\Omega$. On the one hand, if one wants to be sure that the finite elements be accurate enough, it would be better to choose $h$ small enough compared to $H$. On the other hand, such a choice is costly. 
Such an approximation is done in a space which does not move during the optimization algorithm. Now, let $\Omega=\hat{T}_{0}(\hat{\Omega})$ be a set which has been generated by the algorithm. If $\hat{L i p}_{H}$ is spanned by vectors $\left\{\hat{V}_{i}, i=1, . . N\right\}$, the vectors $\left\{V_{i}, i=1, . . N\right\}$ which have to be taken are:

$$
V_{i}=\hat{V}_{i} \circ \hat{T}_{0}^{-1} \quad i=1 . . N
$$

Thus, if $\hat{V}=\sum_{i=1}^{N} \eta_{i} \hat{V}_{i}$ is a given element in $\hat{\operatorname{Lip}}{ }_{H}$, using (32), we get:

$$
\begin{array}{r}
\frac{d j}{d \omega}(\Omega) \cdot V=\frac{d \hat{\jmath}}{d \hat{T}}\left(\hat{T}_{0}\right) \cdot\left(\sum_{i=1}^{N} \eta_{i} \hat{V}_{i}\right) \\
=\sum_{i=1}^{N} \eta_{i} \frac{d \hat{\jmath}}{d \hat{T}}\left(\hat{T}_{0}\right) \cdot \hat{V}_{i} \\
=\sum_{i=1}^{N} \eta_{i} \frac{d j}{d \omega}(\Omega) \cdot V_{i}
\end{array}
$$

so that the discretized gradient is the vector:

$$
\left(G_{i}(\Omega)\right)_{i=1 . . N}=\left(\left[\frac{d j}{d \omega}(\Omega) \cdot V_{i}\right]_{h}\right)_{i=1 . . N}
$$

The computation of $\left[\frac{d j}{d \omega}(\Omega) \cdot V_{i}\right]_{h}$ is done using the formulas given in Proposition 2.1, and approximating of $y^{\Omega}$ and $p^{\Omega}$ by $y_{h}^{\Omega}$ and $p_{h}^{\Omega}$ are described in Section 5.3.1.

\subsection{Implementation}

\subsubsection{Computation of $y_{h}^{\Omega}$ and $p_{h}^{\Omega}$}

The finite element formulations we use are described in Section 5.1. Piecewise linear approximation are used for the partial differential equations, and a piecewise constant one is used to solve the two integral equations. We get $y_{h}^{\Omega}=\left(\psi_{h}^{\Omega}, \theta_{h}^{\Omega}, w_{h}^{\Omega}\right)$ and $p_{h}^{\Omega}=\left(P_{h}^{\Omega}, T_{h}^{\Omega}, q_{h}^{\Omega}\right)$.

Let us describe briefly which algorithms we use in order to get the couple $\left(\theta_{h}^{\Omega}, w_{h}^{\Omega}\right)$. 
Integral equation We use a piecewise constant approximation to solve the integral equation. We denote by $\left\{X_{i}\right\}_{1 \leq i \leq r}$ characteristic functions of surfaces $(\partial \Omega)^{i}$. $\left\{X_{i}\right\}_{1 \leq i \leq r}$ is a base of $\mathcal{W}_{h}(\partial \Omega)$. We seek $w_{h}$ in the space $\mathcal{W}_{h}(\partial \Omega)$ defined as follows: $w_{h}(x)=\sum_{i=1}^{r} w_{h}^{i} X_{i}(x)$. We write:

. $S_{i j}=S_{i} F_{i j}=\int_{(\partial \Omega)^{i}} \int_{(\partial \Omega)^{j}} \phi(x, y) d s(y) d s(x)$.

where $S_{i}$ is the surface of $(\partial \Omega)^{i}$ and $F=\left(F_{i j}\right), 1 \leq i, j \leq r$ is the angle factor matrix (see e.g. Ref. 13). The computation of this matrix is done using a Monte-Carlo method and shadow effects are taken into account. More details concerning its computation can be found in Ref. 16.

. $e_{h}^{i}=\frac{\delta_{1}}{\delta_{2}} \int_{(\partial \Omega)^{i}} \varepsilon \theta_{h}^{4} d s$ (the emittance $\varepsilon$ is assumed to be constant by surfaces).

. $S=\left(S_{i j}\right), 1 \leq i, j \leq r ; \quad E_{h}=\left(\epsilon_{h}^{i}\right)$ and $W_{h}=\left(w_{h}^{i}\right), 1 \leq i \leq r$.

- $D_{\varepsilon}=\operatorname{diag}\left(\varepsilon_{i}\right), 1 \leq i \leq r$

. and $M=\operatorname{diag}\left(S_{i}\right)-\left[I-D_{\varepsilon}\right] S$. We have:

$$
M_{i j}=\mid \begin{array}{lll}
S_{i} & \text { if } & i=j \\
-\left(1-\varepsilon_{i}\right) S_{i j} & \text { if } & i \neq j
\end{array}
$$

The matrix $S$ is symmetric and the matrix $M$ is positive defined. The linear system we solve is :

Given $E_{h}$, find $W_{h} \in \mathbb{R}^{r}$ such that

$M W_{h}=E_{h}$ 
Partial Differential Equation We use a piecewise linear approximation for the partial differential equation and we use a Newton-Raphson's algorithm to linearize the boundary condition. We denote by $\left(V_{0 h}^{\theta}(\Omega)\right)^{\prime}$ the dual space of $V_{0 h}^{\theta}(\Omega)$ and we define the operator $A_{h}^{w}$ as follows:

$$
A_{h}^{w}: V_{t h}^{\theta}(\Omega) \rightarrow\left(V_{0 h}^{\theta}(\Omega)\right)^{\prime}: \theta_{h} \mapsto A_{h}^{w}\left(\theta_{h}\right)
$$

where

$$
\begin{aligned}
<A_{h}^{w}\left(\theta_{h}\right), t_{h}>= & \int_{\Omega} \nabla \theta_{h} \nabla t_{h} d x+P e \int_{\Omega} u_{h} \nabla \theta_{h} t_{h} d x \\
& \quad+\int_{\Gamma_{f}^{\theta}}\left[B i\left(\theta_{h}-\theta_{0}\right)+\frac{\varepsilon}{(1-\varepsilon)}\left(\delta_{1} \theta_{h}^{4}-\delta_{2} w_{h}\right)\right] t_{h} d s \\
= & 0
\end{aligned}
$$

Here, $<,>$ is the scalar product in the duality $\left(V_{0 h}^{\theta}(\Omega)\right)^{\prime}, V_{0 h}^{\theta}(\Omega)$. It is obvious that the operator $A_{h}^{w}$ is differentiable from $V_{t h}^{\theta}(\Omega)$ into $\left(V_{0 h}^{\theta}(\Omega)\right)^{\prime}$. At iterate $n$, we solve the following linear system:

Given $u_{h}, w_{h}$ and $\theta_{h}^{n}$, find $\theta_{h}^{n+1} \in V_{t h}^{\theta}(\Omega)$ such that $\forall t_{h} \in V_{0 h}^{\theta}(\Omega)$, $<\left(A_{h}^{w}\right)^{\prime}\left(\theta_{h}^{n}\right) \cdot \theta_{h}^{n+1}, t_{h}>=<\left(A_{h}^{w}\right)^{\prime}\left(\theta_{h}^{n}\right) \cdot \theta_{h}^{n}, t_{h}>-<A_{h}^{w}\left(\theta_{h}^{n}\right), t_{h}>$

We denote by $\left(t_{i}\right)_{i=1, m}$ a base of $V_{0 h}^{\theta}(\Omega)$ and we seek $\theta_{h}^{n+1}$ in the form:

$\theta_{h}^{n+1}(x)=\sum_{i=1}^{m}\left(\theta_{h}^{n+1}\right)^{i} t_{i}(x)$ ( $m$ is the number of nodes). We denote:

$\Theta_{h}^{n}=\left(\left(\theta_{h}^{n}\right)^{i}\right)_{i=1, m}$

$A_{i j}=\int_{\Omega} \nabla t_{i} \nabla t_{j} d x+\operatorname{Pe} \int_{\Omega} u_{h} \nabla t_{i} t_{j} d x$

$+\int_{\Gamma_{f}^{\theta}}\left[B i+4 \frac{\varepsilon}{(1-\varepsilon)} \delta_{1}\left(\theta_{h}^{n}\right)^{3}\right] t_{i} t_{j} d s$

$B_{j}\left(\Theta_{h}^{n}, W_{h}\right)=\int_{\Gamma_{f}^{\theta}}\left[B i \theta_{0}+\frac{\varepsilon}{(1-\varepsilon)}\left(3 \delta_{1}\left(\theta_{h}^{n}\right)^{4}+\delta_{2} w_{h}\right)\right] t_{j} d s$ 
The linear problem (36) becomes:

Given $W_{h}$ and $\Theta_{h}^{n}$, find $\Theta_{h}^{n+1} \in \mathbb{R}^{m}$ such that $A \cdot \Theta_{h}^{n+1}=B\left(\Theta_{h}^{n}, W_{h}\right)$

The matrix $A$ is sparse, non-symmetric and positive defined.

In order to avoid numerical instabilities, we introduce artificial diffusion into the numerical scheme in a classical manner. We use a streamline diffusion method and the expression of $A_{i j}$ is modified as follows:

$$
\begin{gathered}
A_{i j}=\int_{\Omega}\left(I+\tau_{h}\right) \nabla t_{i} \nabla t_{j} d x+P e \int_{\Omega} u_{h} \nabla t_{i} t_{j} d x \\
+\int_{\Gamma_{f}^{\theta}}\left[B i+4 \frac{\varepsilon}{(1-\varepsilon)} \delta_{1}\left(\theta_{h}^{n}\right)^{3}\right] t_{i} t_{j} d s
\end{gathered}
$$

where $\tau_{h}$ is the matrix of artificial diffusion. It is positive defined and its terms vanish with $h$. Its expression is: $\tau_{h}=\frac{h}{2}(P e)^{2} u_{h}{ }^{T} u_{h}$.

Algorithm of Solution In order to solve Problem $\left(P_{h}^{\theta}\right)$, we use the following algorithm of relaxation:

a. Initialization: $\Theta_{h}^{0}$ given.

b. Let $\Theta_{h}^{n}$ known on $\partial \Omega$, compute $W_{h}^{n}$ solution of (35).

c. Let $W_{h}^{n}$ and $\Theta_{h}^{n}$, compute $\Theta_{h}^{n+1}$ solution of (37).

d. Test: if $\frac{\left\|\Theta_{h}^{n+1}-\Theta_{h}^{n}\right\|}{\left\|\Theta_{h}^{n}\right\|}<\varepsilon$ then stop. If not, re-iterate.

We do not study the convergence of this algorithm. Nevertheless, we never had any problem in practice concerning its convergence. 


\subsubsection{Gradient Computation}

In our problem, the shape of $\Omega$ is governed by the shape of $\Gamma_{\text {hose }}$. We have modeled it in a classical manner. We consider coordinates $(\xi, \eta)$ in $\mathbb{R}^{2}$ (see Fig. 6).

Shape of $\Gamma_{\text {hose }}$ and control parameters. For a sake of simplicity, the hose is supposed to have a constant thickness $\epsilon$ along the $\eta$ axis. One side is the graph of a $C^{2}$ function $\eta \mapsto \eta(\xi):[a, b] \rightarrow \mathbb{R}$. The other one is its translated. We approximate the function using cubic splines. We consider a discretization $a<\xi_{1}<\xi_{2} \ldots<\xi_{N}<$ $b$ of $[a, b]$ and $\left\{s_{i}(\xi), i=1 . . N\right\}$ the corresponding basis of the finite dimensional vector space of one dimensional cubic splines defined by:

$$
s_{i}\left(\xi_{j}\right)=\delta_{i j} \quad i=1 . . N, j=1 . . N
$$

(here for the sake of simplicity we have chosen $h=H$ ).

The shape of a hose is given by a function:

$$
\eta_{h}(\xi)=\sum_{i=1}^{N} \eta_{i} s_{i}(\xi)
$$

and we have $\eta_{h}\left(\xi_{i}\right)=\eta_{i}$. (We could as well have chosen the basis made of B-splines. The unknown coefficients would not correspond directly to the position of nodes). As the shape $\Omega$ is completely determined by the shape of $\Gamma_{\text {hose }}$, the control parameters are the $N$ values $\left\{\eta_{i}, i=1 . . N\right\}$.

Construction of $\hat{V}_{i}$ and $V_{i}$. We consider a reference shape. For instance, we take a straight hose so that:

$$
\hat{\Gamma}_{\text {hose }}=\{(\xi, \eta), \xi \in[a, b], \eta=0\} \cup\{(\xi, \eta), \xi \in[a, b], \eta=e\}
$$


For each $i \in\{1, . ., N\}$ we choose $\hat{V}_{i} \in \operatorname{Lip}\left(\hat{\Omega} ; \mathbb{R}^{2}\right)$ solution of:

$$
\begin{aligned}
& \Delta \hat{V}_{i}(x)=0 \quad \text { in } \hat{\Omega} \\
& \hat{V}_{i}(x)=\left(0, s_{i}(\xi)\right) \quad \text { on } \hat{\Gamma}_{\text {hose }} \\
& \hat{V}_{i}(x)=0 \quad \text { on } \partial \hat{\Omega} \backslash \hat{\Gamma}_{\text {hose }}
\end{aligned}
$$

(in this manner, the nodes which are on the hose can move in the horizontal direction only, the end points of the hose do not move). The space $\hat{L i p}_{H}$ is spanned by these vectors $\left\{\hat{V}_{i}, i=1 . . N\right\}$.

Now, if the optimization algorithm has brought us to a shape $\Omega_{n}=\hat{T}_{0}^{n}(\hat{\Omega})$, according to (32) we consider:

$$
V_{i}^{n}=\hat{V}_{i} \circ\left(\hat{T}_{0}^{-1}\right)^{n} \quad i=1 . . N
$$

These are easy to compute. The vector fields just follow the movement of $\Omega$ from $\Omega_{0}$ to $\Omega_{n}$ in the algorithm. $V_{i}^{n}$ and $\hat{V}_{i}$ have the same value at the corresponding points.

Then, it is straightforward to compute:

$$
G_{i}\left(\Omega_{n}\right)=\left[\frac{d j}{d \omega}(\Omega) \cdot V_{i}^{n}\right]_{h} \quad i=1 . . N
$$

given by (33).

Optimization algorithm. We describe how the algorithm builds $\Omega_{n+1}$ from $\Omega_{n}$. $\Omega_{n}$ is characterized by the coefficients $\left\{\eta_{i}^{n}, i=1 . . N\right\}$ which give the position of $\Gamma_{\text {hose }}^{n}$. Previously, we have seen how to compute the approximation $\left[j\left(\Omega_{n}\right)\right]_{h}$ of $j\left(\Omega_{n}\right)$ and how to compute the gradient $G_{i}\left(\Omega_{n}\right)=\left[\frac{d j}{d \omega}\left(\Omega_{n}\right) \cdot V_{i}^{n}\right]_{h}, i=1 . . N$. Then we use a descent method. Namely, we have tested the method with a BFGS algorithm for 
the choice of the descent direction, and a Wolfe's law for the line search. Notice that if we would have used the gradient method, we would have chosen:

$$
\eta_{i}^{n+1}=\eta_{i}^{n}-\rho_{n} G_{i}\left(\Omega_{n}\right) \quad i=1 . . N
$$

where $\rho_{n}$ has to be adjusted.

Remark 5.1 The functional $j(\Omega)$ depends on $\Omega$ through $\left(\eta_{1}, . ., \eta_{N}\right)$ only. If we denote:

$$
\mathcal{J}\left(\eta_{1}, . ., \eta_{N}\right)=j(\Omega)
$$

so that the minimization algorithm can be written:

$\left(\eta_{1}^{n}, . ., \eta_{N}^{n}\right), \mathcal{J}\left(\eta^{n}\right), G\left(\Omega_{n}\right)$ are given, Compute $\eta^{n+1}$ such that $\eta^{n+1}=\eta^{n}-\rho^{n} d^{n}$

where $d^{n}$ is the direction of descent and $\rho^{n}>0$ is the step of descent.

The optimization procedure can be schematized on Figure 5.

The software we have used has been written in three steps. A. Habbal, working for the companies SIMULOG S.A. and RENAULT S.A. has written the optimization process without radiation. The MODULEF and MODULOPT libraries have been used. The angle factors $\phi(x, y)$ are computed by a software using a Monte-Carlo's method and shadow effects are taken into account. This last software has been written by A. Mezrhab in his thesis Ref. 16. Finally, J. Monnier has completed these softwares in order to incorporate the radiation effects into the optimization process. 


\section{Numerical Results}

Let us recall that we consider an air flow under a car hood and parts under the hood are schematized as indicated on Figure 4. We refer to Section 3 for a description of the physical model.

Let us recall that the goal of the following numerical results is to prove that we are able to solve the initial industrial problem using the analysis and technic developed in the present paper. It is a feasibility study. The software we developed (which is already rather big), must be much more developed in order to obtain some interesting results in an industrial and engineering point of view.

Physical data of the model correspond to a car moving at 80 kilometers per hour.

We have $\rho=1 \mathrm{~kg} / \mathrm{m}^{3}, C_{p}=1010 \mathrm{~J} /(\mathrm{kg} . \mathrm{K}), \lambda=310^{-2} \mathrm{~W} /(\mathrm{m} . \mathrm{K})$ and the Peclet number Pe equals $10^{5}$. Let us notice that the thermal conductivity $\lambda$ of the air is small and the assumption on $\lambda$ in Proposition 4.1 certainly does not hold in our case. So, is the temperature differentiable with respect to the domain? Hence, is the cost function differentiable with respect to the domain in our case? The only thing we can affirm is that in practice we did not meet any special difficulties.

Physical data on the boundary are the following. The normal velocity of the flow at the air entrance (i.e. on the radiator exit) equals $5 \mathrm{~m} / \mathrm{s}$. The fluid potential is known at the exit. The condition $u . n$ equals 0 is imposed everywhere else on the boundary. Temperatures are imposed on the air entrance $\left(280^{\circ} \mathrm{K}\right)$, on the engine block $\left(420^{\circ} \mathrm{K}\right)$ and on the exhaust pipe $\left(520^{\circ} \mathrm{K}\right)$. We consider on the hose, on the 
screen, on the battery and on the external surface, heat transfer by radiation and convection described by (18) with the following data:

. on the hose, $\theta_{0}=300 \mathrm{~K}, h=10 \mathrm{~W} /\left(m^{2} \mathrm{~K}\right)$ ( $h$ being the thermal transfer coefficient) and $\varepsilon=0.5$.

. on the screen, $\theta_{0}=330 \mathrm{~K}, h=10 \mathrm{~W} /\left(m^{2} \mathrm{~K}\right)$ and $\varepsilon=0.5$.

. on the battery, $\theta_{0}=330 \mathrm{~K}, h=10 \mathrm{~W} /\left(\mathrm{m}^{2} \mathrm{~K}\right)$ and $\varepsilon=0.5$.

. on the external surfaces, $\theta_{0}=290 \mathrm{~K}, h=20 \mathrm{~W} /\left(m^{2} \mathrm{~K}\right)$ and $\varepsilon=0.5$.

Concerning the air exit, we assume that the thermal flows $\nabla \theta . n$ equals 0 and in a radiative point of view, we model it as a black body at the temperature $\theta_{\text {exit }}=320 \mathrm{~K}$

Finally, the emittance value of the radiator (i.e. the air entrance) and of the exhaust pipe is $\varepsilon=0.5$ and the emittance value of the engine block is $\varepsilon=0.8$.

In order to validate the simulator of the software (i.e. the state equation and the adjoint state equation), we used exact solutions, we plotted the error graphs as a function of the mesh size and we obtained lines with the expected slopes. Then, in order to validate the gradient values, we compared values of the discretized continuous gradient computed by this software with values obtained using a finite difference method. The results are comparable and can be found in Ref. 11.

We consider the following two initial shapes: shape a (Figs. 7 and 8) and shape b (Fig. 9). We seek an optimal shape from these two different initial shapes 
and we obtained the same "optimal" shape (Fig. 10).

Let us notice that the cost function depends on the hose temperature and on its length as well. In others respects, the fluid velocity printed on the figures is not exactly the finite element solution (i.e. $\nabla \psi_{h}$ ). Indeed, the field is obtained by a linear interpolation of the potential $\psi_{h}$.

Let us precise that we performed others computations than those presented below (see Ref. 11) and for every computation we obtained the same "optimal" shape from few different initial shapes and with different numbers of points of splines. (We tested with different numbers of points of splines between 4 and 8 ). So, is this "optimal" shape a local minimum or a global minimum? We don't know it. But, the fact that we obtained each time the same "optimal" shape independently of the initial shape and independently of the number of points of splines, in addition with a substantial cost benefit, is already an interesting result.

We present in tables 1 and 2 , the cost function values and the gradient values, initial and optimal, computed from the two different shapes a and b. The normalized gradient which is present is the norm 1a of the gradient divided by a characteristic value of the cost function.

Starting from shape a, the algorithm stopped because it was unable to find a better shape. Starting from shape b, the algorithm stopped because the cost benefit between two iterates was very small. In both cases, the algorithm had converged. 
Let us point out that if the initial shape is very different than the "optimal" one (like shape b), we necessarily need to re-mesh the geometry, in others words to restart a process of optimization. With our software, this re-meshing procedure is done automatically. And, in order to obtain the "optimal" shape from shape b, it has been necessary to re-mesh twice. 


\section{References}

1. MONNIER, J., and VILA, J.P., Convective and Radiative Thermal Transfer with Multiple Reflections. Analysis and Approximation by a Finite Element Method, Mathematical Models and Methods in Applied Sciences, Vol. 11, 2001.

2. BREZIS, H., Analyse Fonctionnelle: Théorie et Applications, Masson, Paris, France, 1983.

3. MURAT, F., and SIMON, J., Sur le Contrôle par un Domaine Géométrique, Publication of the Laboratory of Numerical Analysis, University Paris VI, 1976.

4. CHEnAIS, D., Cours de 3ème Cycle, University of Nice-Sophia Antipolis, 1991.

5. CÉA, J., Conception Optimale ou Identification de Formes. Calcul Rapide de la Dérivée Directionnelle de $\quad$ la Fonction Coût, $\quad$ Modélisation Mathématique et Analyse Numérique, Vol. 20, pp 371-402, 1986.

6. ROUSSELET, B., Quelques Résultats en Optimisation de Domaine, Thèse d'État, University of Nice, 1982.

7. PIRONNEAU, O., Shape Optimum Design for Elliptic Systems, Springer Verlag, New York, NY, 1984. 
8. MASMOUDI, M., Outils pour la Conception Optimale de Formes, Thèse d'État, University of Nice, 1987.

9. SOKOLOWSKI, J., and ZOLESIO, J.P., Introduction to Shape Optimization: Shape Sensitivity Analysis, Springer Verlag, Berlin, Germany, 1992.

10. Guillaume, P., Dérivées d'Ordre Supérieur en Conception Optimale de Forme, Thèse de 3ème Cycle, University of Toulouse, 1994.

11. MONNIER, J., Conception Optimale de Forme pour un Système Couplé Fluide-Thermique. Application à l'Aérothermique d'un Véhicule, Thèse de 3ème Cycle, University of Nice-Sophia Antipolis, 1995.

12. HAUG, E.J., CHOI, K.K., KOMKOV, V, Design Sensitivity Analysis of Structural Systems, Mathematics in Sciences and Engineering, Academic Press, New York, NY, vol. 177, 1986.

13. SPARROW, E.M., and CESS, R.D., Radiation Heat Transfer, Mc Graw-Hill Book, New York, NY, 1978.

14. GRISVARD, P., Elliptic Problems in Non Smooth Domains, Monograph and Studies in Mathematics, Pitman, London, England, Vol. 24, 1985.

15. PERRET, C., and WITOMSKI, P., Équation de la Chaleur et Réflections Multiples, Annales de l'Institut Poincaré, Vol. 8, pp 677-689, 1991.

16. MEZRHAB A., Contribution à l'Étude Numérique et Expérimentale des Échanges Radiatifs, Thèse de 3ème Cycle, University of Provence, 1991. 
17. MONNIER, J., Shape Sensitivity in a Navier-Stokes Flow with Radiative Thermal Transfer, to appear. 


\section{List of Tables}

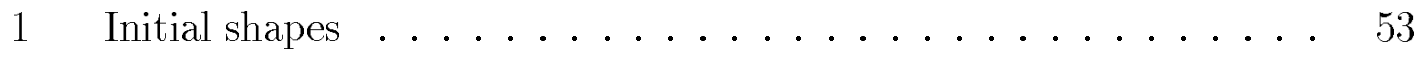

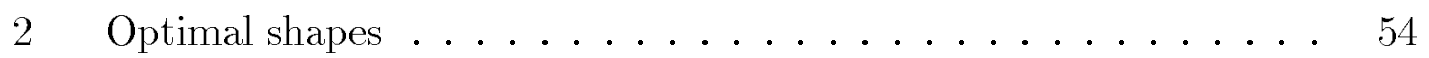


Table 1: Initial shapes

\begin{tabular}{|c|c|c|c|}
\hline \multicolumn{4}{|c|}{ Initial shapes } \\
\hline Shape & Normalized gradient & Cost & Temp. max. hose \\
\hline \hline $\mathrm{a}$ & 1.45 & 62534 & $121{ }^{\circ} \mathrm{C}$ \\
\hline $\mathrm{b}$ & 4.2 & 72827 & $139{ }^{\circ} \mathrm{C}$ \\
\hline
\end{tabular}


Table 2: Optimal shapes

\begin{tabular}{|c|c|c|c|c|}
\hline \multicolumn{5}{|c|}{ Optimal shapes } \\
\hline Shape & Normalized gradient & Cost & Gain & Temp. max. hose \\
\hline \hline $\mathrm{a}$ & 0.54 & 62380 & $0.3 \%$ & $120{ }^{\circ} \mathrm{C}$ \\
\hline $\mathrm{b}$ & 0.58 & 60539 & $17 \%$ & $120{ }^{\circ} \mathrm{C}$ \\
\hline
\end{tabular}




\section{List of Figures}

1 Example of a non Bi-lipschitz homeomorphism . . . . . . . 56

2 Change of variables . . . . . . . . . . . . 57

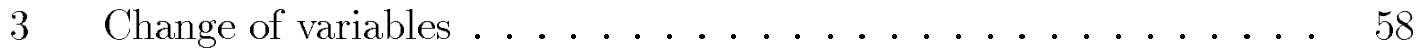

4 Air flow under a car hood . . . . . . . . . . . . . . 59

5 Optimization process .................... 60

6 Discretization of the hose . . . . . . . . . . 61

$7 \quad$ Initial shape (a). Mesh . . . . . . . . . . . . . 62

8 Initial shape (a). Isotherms . . . . . . . . . . 63

9 Initial shape (b). Mesh . . . . . . . . . . . . . . 64

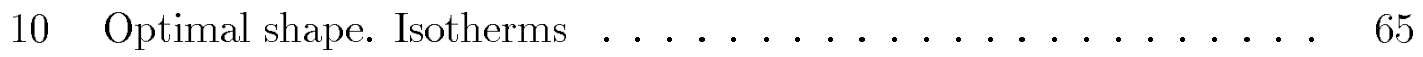



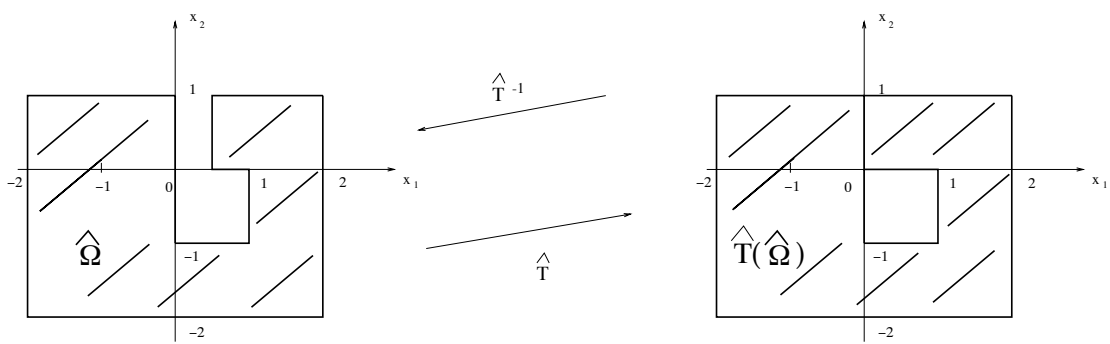

Figure 1: Example of a non Bi-lipschitz homeomorphism 


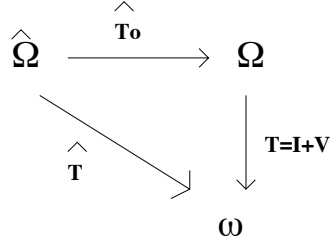

Figure 2: Change of variables 


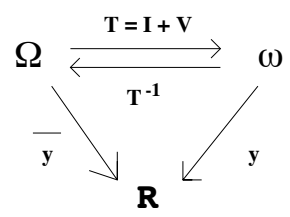

Figure 3: Change of variables 


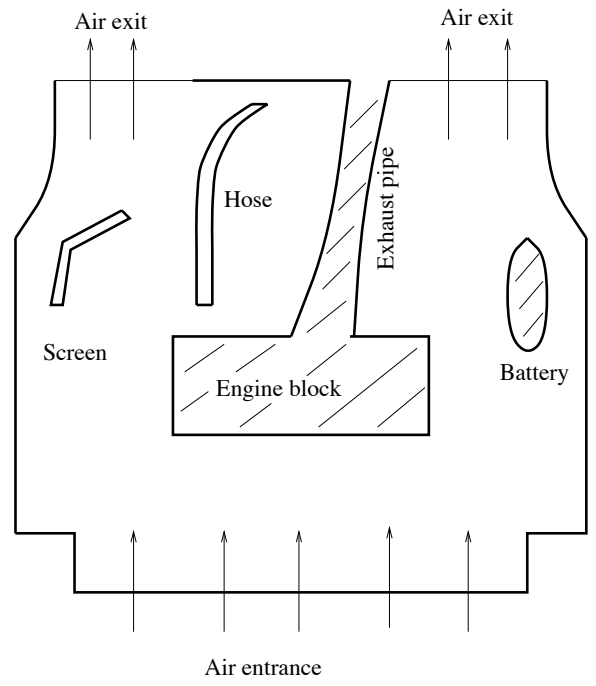

Figure 4: Air flow under a car hood 


\begin{tabular}{|c|c|c|}
\hline SIMULATOR & 7 & \\
\hline \multicolumn{3}{|l|}{ DIRECT PB (NON LINEAR) } \\
\hline \multicolumn{3}{|l|}{ | FLUID PDE } \\
\hline \multicolumn{3}{|l|}{ THERMAL PDE } \\
\hline \multicolumn{3}{|l|}{ INTEGRAL EQN } \\
\hline$y_{h}=\left(\psi_{h} \theta_{h} w_{h}\right)$ & & OPTIMIZER \\
\hline COST FUNCTION & & ALGORITHM OF \\
\hline $\mathrm{J}_{\mathrm{h}}\left(\Omega_{\mathrm{n}}\right)=\ldots$ & LINE SEARCH. & DESCENT: BFGS \\
\hline \multicolumn{3}{|l|}{ ADJOINT PB (LINEAR) } \\
\hline \multicolumn{3}{|l|}{ ADJOINT INTEGRAL EQN } \\
\hline \multicolumn{3}{|l|}{ ADJOINT THERMAL PDE } \\
\hline \multicolumn{3}{|l|}{ ADJOINT FLUID PDE } \\
\hline \multicolumn{3}{|l|}{$\mathrm{p}_{\mathrm{h}}=\left(\mathrm{P}_{\mathrm{h}}, \mathrm{T}_{\mathrm{h}}, \mathrm{q}_{\mathrm{h}}\right)$} \\
\hline \multicolumn{3}{|l|}{ GRADIENT } \\
\hline \multicolumn{3}{|l|}{$\mathrm{G}\left(\Omega_{\mathrm{n}}\right)=\ldots$} \\
\hline
\end{tabular}

Figure 5: Optimization process 


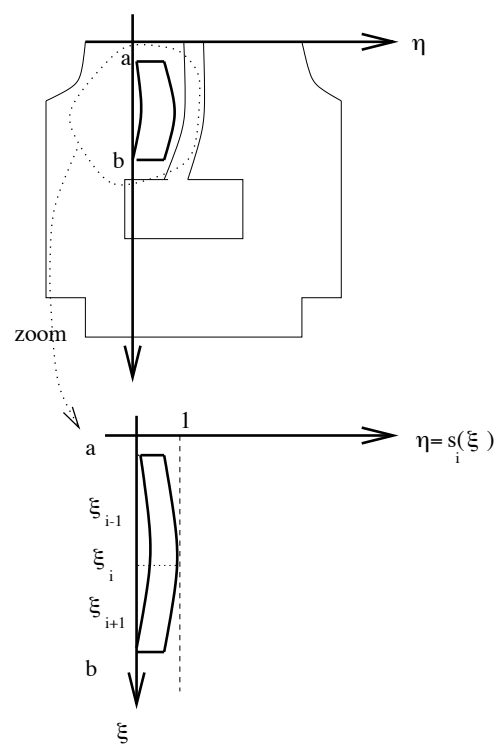

Figure 6: Discretization of the hose 


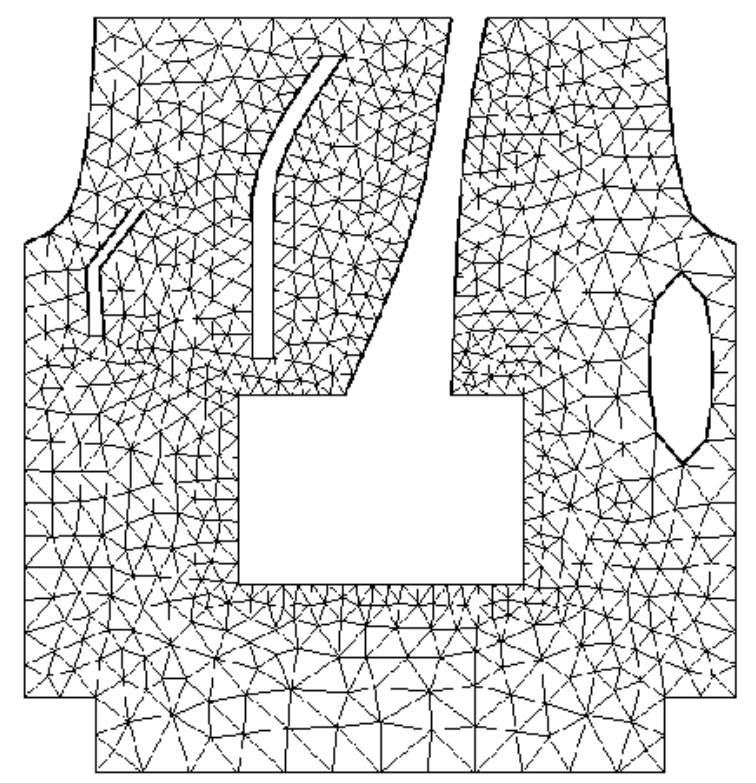

Figure 7: Initial shape (a). Mesh 


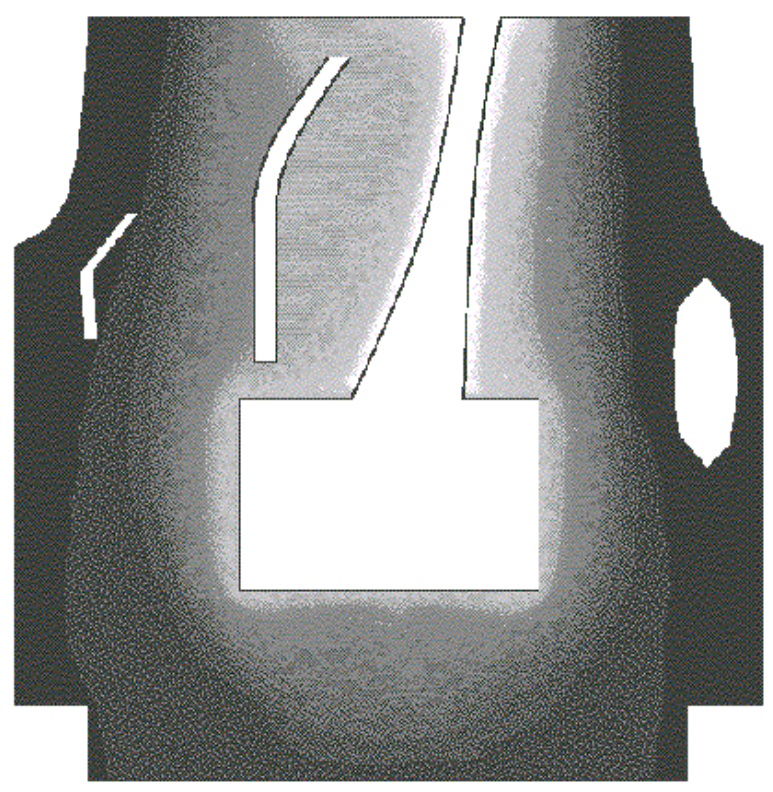

Figure 8: Initial shape (a). Isotherms 


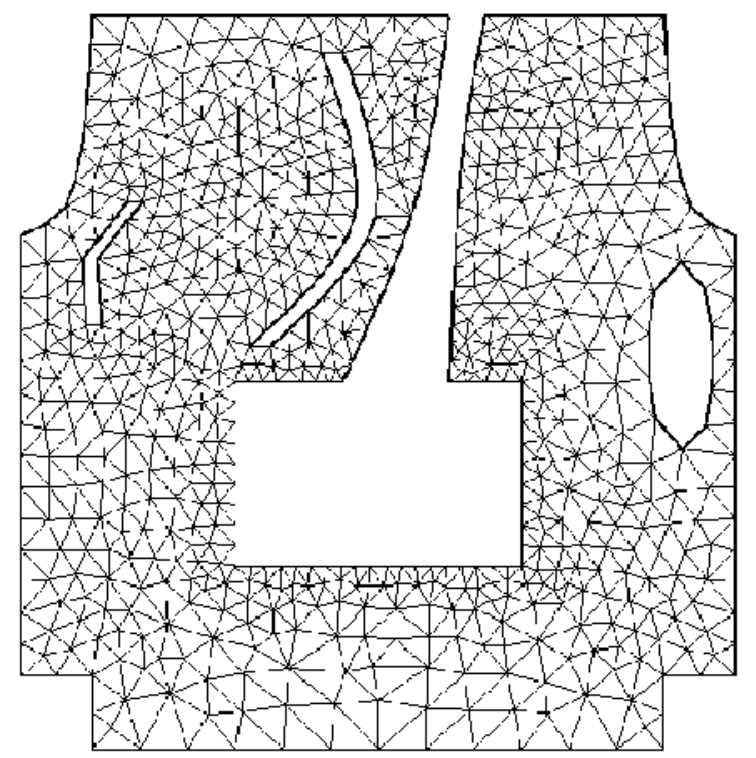

Figure 9: Initial shape (b). Mesh 


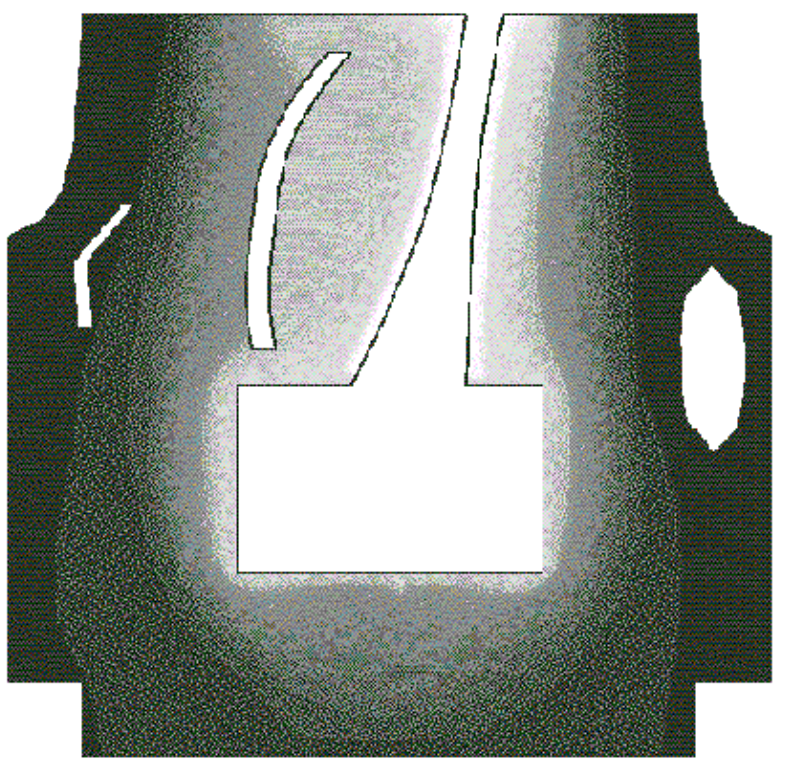

Figure 10: Optimal shape. Isotherms 